\title{
In-depth characterization of icosahedral ordering in liquid copper
}

\author{
Szymon Winczewski ${ }^{\mathrm{a}}$, Jacek Dziedzic ${ }^{\mathrm{a}, \mathrm{b}}$, Jarosław Rybicki ${ }^{\mathrm{a}, \mathrm{c}}$ \\ ${ }^{a}$ Faculty of Applied Physics and Mathematics, Gdansk University of Technology, \\ Narutowicza 11/12, 80-233 Gdańsk, Poland. \\ ${ }^{b}$ Department of Chemistry, University of Southampton, \\ Highfield, Southampton SO17 1BJ, United Kingdom \\ cTASK Computer Centre, Gdansk University of Technology, \\ Narutowicza 11/12, 80-233 Gdańsk, Poland
}

\begin{abstract}
The presence of icosahedral ordering in liquid copper at temperatures close to the melting point is now well-established both experimentally and through computer simulation. However, a more elaborate analysis of local icosahedral and icosahedron-like structures, together with a system for classifying such structures based on some measure of "icosahedrity", has so far been conspicuously absent in the literature. Similarly, the dynamics of these structures has not yet received the attention it merits.

We present a new method for structural analysis, which combines Voronoi analysis with bond-orientational order parameters, and apply it to liquid $\mathrm{Cu}$ configurations obtained from tight-binding molecular dynamics at a range of temperatures near the melting point. We introduce a clear system for classifying local structures according to their degree of similarity to the perfect icosahedron, and show how their energies of formation correlate with our structural descriptor. We examine the frequencies of occurrence for the classes of Voronoi polyhedra we distinguish, calculate their lifetimes, and establish the temperature dependence of these properties. We explore the dynamics of icosahedron-like structures by examining how individual classes transform between one another. Finally, we perform structural correlation analysis, demonstrating, among other things, that icosahedra and icosahedron-like structures preferentially connect and show a tendency towards clustering. We believe our approach can be readily applied in studies of icosahedral ordering in liquid metals or metallic glasses.
\end{abstract}

Keywords: liquid copper, icosahedral ordering, Voronoi analysis, bond-orientational order parameters, tight-binding molecular dynamics

\section{Introduction}

The structure of a liquid is often said to be "random", but this is not the case. While it is true that long-range order is absent, atomic positions in a liquid remain significantly correlated in the short-range. These correlations engender preference for certain structural motifs, which then occur more often, and can thus be characterized.

The concept of icosahedral ordering, introduced by Frank in 1952 [1], plays a central role in descriptions of the structure of liquid metallic systems. In his theoretical analyses of small (13-atom) clusters, Frank recognized that icosahedral clusters have energies that are about $8 \%$ lower than for clusters corresponding to close-packed fcc and hcp structures. He theorized that the presence of icosahedra stabilizes the liquid structure, which could explain significant undercooling (up to 15-25\% of the melting temperature for certain elements and compounds) that had by then been observed experimentally [2, 3].

It took almost three decades, and major advances in computational methods and capabilities, for Frank's hypothesis to be confirmed. In the $1980 \mathrm{~s}$ the presence of icosahedral ordering has been demonstrated in

Email address: wisnia@kdm.task.gda.pl (Szymon Winczewski) 
molecular dynamics (MD) simulations for a number of simple model systems, such as monoatomic [4-7] and binary [7] Lennard-Jones liquids.

Subsequently, owing to progress in diffraction methods, Frank's hypothesis was also confirmed on experimental grounds. In 2002, through neutron scattering and electromagnetic levitation, Schenk et al. [8] detected icosahedral ordering in significantly undercooled liquid metals such as $\mathrm{Ni}, \mathrm{Fe}$ and $\mathrm{Zr}$. A year later Di Cicco and coworkers, using X-ray absorption spectroscopy together with reverse Monte Carlo, convincingly demonstrated that icosahedral ordering is also present in undercooled liquid $\mathrm{Cu}$, and in liquid $\mathrm{Cu}$ above the melting point [9].

Liquid copper has been a subject of computational studies since the late 1980s. Initial research focused on assessing the quality of description yielded by available interaction models: effective pair potential [10, 11], embedded atom method (EAM) [12-17], density functional theory (DFT) [18]. Encouragingly, it turned out to be possible to obtain a credible description of the liquid $\mathrm{Cu}$ system, with reasonable agreement with experiment for crucial structural properties (structure factor [11, 12], pair correlation function [11, 13, 15, 16, 18]), thermodynamical properties (specific heat $[11,14,16]$, melting temperature [10, 13, 15, 16], coefficient of thermal expansion [13, 14, 16], Gibbs free energy [10, 15]), and transport properties (selfdiffusion coefficient $[11,13,17,18]$, viscosity coefficient $[11,17])$.

Following the experimental discovery of Di Cicco et al. [9] subsequent computational studies of liquid copper mostly focused on icosahedral ordering. Later work by Di Cicco and his coworkers [19-21], together with a paper by Ganesh and Widom [22], further strengthened the initial experimental findings of Ref. [9], demonstrating (this time on computational grounds), that icosahedral ordering is indeed present in liquid and undercooled-liquid copper.

The quantitative characterization of icosahedral ordering undertaken in Refs. [19-22] yielded results in good agreement with the experimental estimate of Ref. [9] that predicted icosahedron-like structures to comprise up to $10 \%$ of all structural units in liquid $\mathrm{Cu}$. The analysis of energetics carried out in Ref. [20] demonstrated that the presence of icosahedra - both perfect and defected - indeed stabilizes the structure of liquid copper, hindering crystallization, which directly confirmed Frank's hypothesis.

While the above-mentioned papers provided strong evidence for the presence of icosahedral ordering in liquid $\mathrm{Cu}$, a more elaborate analysis of its structural details is still missing in the literature. In particular, no papers set out to identify or classify the types of icosahedral and icosahedron-like structures, or to quantify their degree of what we shall term "icosahedrity", i.e., how similar a given local structure is to a perfect icosahedron. Introducing such classification would enable a better understanding of the roles played by individual local structures in the global picture of icosahedral ordering, helping to elucidate how the specifics of these local structures translate into the properties of the system under study.

In this work we tackle the above problem, setting out to perform an elaborate characterization of icosahedral ordering in liquid copper, continuing and extending the research lines established in Refs. [9, 19-22].

This paper is organized as follows. In Sec. 2 we describe the protocol we followed in our tight-binding molecular dynamics (TB-MD) simulations of liquid copper. Sec. 3 presents the new approach for structural analysis that we developed for the purposes of this work. This approach successfully combines two commonly used methods - Voronoi analysis [23] and the bond-orientational order parameters method [4, 5]. Section 4 is devoted to results. We begin with a glance at mean structural measures, ensuring that the overall structural picture of liquid $\mathrm{Cu}$ obtained from our simulations is consistent with the experimental picture of Ref. [9] and first-principles calculations of Ref. [22]. We then present the results of our elaborate analysis of icosahedral ordering, first clearly identifying structures that comprise it in liquid $\mathrm{Cu}$, then, by examining their lifetimes and the transformations they undergo, we establish the roles they play in the dynamical structural picture of this system. We finish with a characterization of medium-range order, ascertaining that icosahedron-like structures in liquid $\mathrm{Cu}$ display a tendency to cluster that is often observed in other systems. Sec. 5 contains discussion. We confront our picture of ordering in liquid $\mathrm{Cu}$ with the recent results for liquid Fe due to Pan et al. [24], followed by a short critique of common neighbor analysis, a method sometimes used to study icosahedral ordering. Sec. 6 presents brief conclusions. 


\section{Computational details}

All simulations in this work were carried out using tight-binding molecular dynamics (TB-MD). Tightbinding (TB) approaches provide a qualitative description of electronic effects that can be viewed as a stationary approximation to self-consistent density functional theory (DFT) [25]. The Born-Oppenheimer approximation is invoked to move the classically treated atomic ions in response to Hellmann-Feynman forces obtained following a diagonalization of the electronic Hamiltonian. Pulay contributions [26] to forces are absent (vanish identically) [27] in this approach.

The Naval Research Laboratory total energy tight-binding formulation [28-34] (NRL TB) was used. NRL TB is a non-orthogonal, non-charge-self-consistent, two-center variant of tight binding. Its distinguishing feature is that the diagonal on-site terms in the tight-binding Hamiltonian are not constants, but instead depend on the atom's generalized local density [35]. This improves transferability and eliminates what is known as a residual energy term $[25,36]$ from the total energy expression. For $\mathrm{Cu}$ in particular the numerical accuracy of forces and energies obtained from NRL TB was shown to compare favorably against plane-wave DFT [37, 38], including for phases not included in the fit, which is a testament to the method's transferability. While a generalized diagonalization of the Hamiltonian is necessary to compute TB energies and forces, making the method cubic-scaling, its computational simplicity translates into a performance gain of 2-3 orders of magnitude compared to DFT calculations [35], enabling dynamical calculations on hundreds of atoms over a picosecond time scale.

We sampled a range of temperatures between $1300 \mathrm{~K}$ and $1900 \mathrm{~K}$ in increments of $100 \mathrm{~K}$ (the experimental melting point of bulk $\mathrm{Cu}$ being $1358 \mathrm{~K}$ ). Each system comprised 500 atoms, arranged in an fcc lattice with densities corresponding to experimental values for liquid $\mathrm{Cu}$ taken from Ref. [39]. Interactions were cut off smoothly at $r_{\text {cut }}=12.5 a_{0}$ ( $a_{0}$ being the Bohr radius). Details of the NRL TB cutoff function can be found in Ref. [40]. To improve sampling, seven independent runs with different initial velocities were performed for each of the temperatures, and all results reported in this work are averages over these runs. Simulations were carried out in the NVT ensemble with the temperature controlled using a Nosé-Hoover [41-43] thermostat. Internal pressures were monitored and found not to exceed $10 \mathrm{GPa}$, and average temperatures were within $4 \mathrm{~K}$ from desired values for each run. A time step of $\Delta t=0.5 \mathrm{fs}$ was used. Equations of motion were integrated using a Gear predictor-corrector [44] method. Each simulation began with an equilibration stage, where velocity scaling was employed for the first 1000 steps, followed by 4000 steps of thermostatting. Production calculations ran for 10000 steps (5 ps). While our timescales were necessarily limited, the use of seven independent runs greatly improves sampling and enables rudimentary error estimates.

\section{Structural analysis}

A number of methods are commonly employed in structural analysis of disordered systems. One commonly used approach is Voronoi analysis [23] (examples of use in Refs. [24, 45-53]). Other typical choices include bond-orientational order parameters [4, 5] (BOPs) (examples in Refs. [9, 19, 20, 22, 48, 54]), the Honeycutt-Andersen index [55] (HA) (examples of use can be found in Refs. [22, 45, 48, 56-59]), and its extension, common-neighbor analysis [60, 61] (CNA) (examples of use in Refs. [20, 21]).

\subsection{Voronoi analysis}

The Voronoi method [23] gives means for analyzing short-range order (SRO). It begins by constructing a Voronoi diagram, whereby coordination polyhedra defined by bisector planes are determined for all particles. Local ordering is inferred from geometrical properties of the obtained polyhedra. To this effect each polyhedron (corresponding to particle $i$ ) is assigned a signature, which is a vector $\left(f_{3}(i), f_{4}(i), f_{5}(i), f_{6}(i)\right)$, where $f_{e}(i)$ denotes the number of faces with $e$ edges. The type of packing is deduced by comparing obtained signatures against reference signatures, such as $(0,12,0,0)$ for fcc packing or $(0,0,12,0)$ for a perfect icosahedron.

When applied to liquid systems, Voronoi analysis runs into difficulties. The disorder is mirrored in the Voronoi diagram, leading to polyhedra with considerably different signatures. Further complications arise due to the thermal motions of the atoms, which bring about polyhedra with a multitude of small faces and 
short edges. Both of these issues hinder analysis, with typical (raw, unprocessed) Voronoi diagrams often spanning thousands of different signatures.

Methods for working around the aforementioned difficulties, at least to a degree, have been proposed. Small faces are commonly filtered out from the polyhedra [62,63], with the criterion for elimination being the ratio of the area of the face to its average value (in the same polyhedron or in the entire diagram). Although such filtering helps with interpretation, it needs to be undertaken carefully, as overzealous filtering distorts the picture.

Intepretational difficulties plaguing liquid systems can, in principle, be countered differently, through a determination of so-called inherent structures [64, 65]. In this approach energy minimization is performed individually for each snapshot obtained from a simulation, and structural analysis is performed for local potential energy minima thusly identified. In this way the distortions due to thermal motions can be filtered out from the diagram, elucidating basic structural motifs present in the liquid.

Unfortunately, energy minimization of liquid structures often runs into practical difficulties related to the fact that their potential energy surface is fraught with multiple minima. This problem is even seen in liquid systems described by simple pair potentials [64]. Moreover, as demonstrated in Ref. [64] the resultant structural picture is sensitive to the method used for minimizing the energy. For more complex and computationally demanding descriptions of interactions, such as tight binding, obtaining inherent structures becomes infeasible.

It is worth pointing out that a new approach has recently been proposed for addressing the difficulties resulting from large thermal motions. The polyhedral template matching (PTM) [66] method simultaneously uses a Voronoi tesselation and a (dual) Delaunay triangulation to effectively classify local structures, even at temperatures slightly below the melting temperature. However, there are no mentions to date of this approach being used for elaborate analysis of the structure of liquid systems. We believe this stems from the fact that the use of PTM necessitates specifying the sought reference structures, which may prove problematic for liquids, owing to the multiplicity, diversity and irregularity of local structures present in them.

Being able to capture the information on the topology of coordination in a compact signature $\left(f_{3}(i), f_{4}(i), f_{5}(i), f_{6}(i)\right)$ is an advantage of Voronoi analysis. This very compactness, however, can also be seen as a disadvantage - the signature is missing substantial information on the symmetries of the polyhedron it describes. For instance, it is possible that the shapes of two polyhedra with the same signature differ markedly. With this in mind, it is prudent to augment Voronoi analysis with a technique that is sensitive to symmetries.

\subsection{Bond-orientational order parameters}

Bond-orientational order parameters (BOPs) were proposed by Steinhardt et al. in 1981-1983, initially for studying orientational order in liquids and glasses $[4,5]$. Over the years the BOP method gained popularity and today it is a staple tool in structural analysis of condensed matter. It is used for characterizing solids [6769], liquids [9, 19, 20, 22, 48, 54, 69], and for studying crystallization [70-72] and melting [73-75].

Within the BOP framework short-range order is studied by calculating two real-valued parameters $Q_{l}(i)$ and $\hat{W}_{l}(i)$ for every particle $i$. First, the set of nearest neighbors $\mathcal{B}(i)$ (with a cardinality $N_{\mathcal{B}(i)}$ ), i.e., the set of all atoms $j \neq i$, where $r_{i j} \leq r_{\mathrm{c}}$, is determined for each particle. In so doing, bonds $\mathbf{r}_{i j}=\mathbf{r}_{j}-\mathbf{r}_{i}$, where $j \in \mathcal{B}(i)$, and $\mathbf{r}_{i}$ is the position of atom $i$, are determined. The bonds are then projected onto the unit sphere, yielding spherical coordinates $\theta\left(\mathbf{r}_{i j}\right), \phi\left(\mathbf{r}_{i j}\right)$. Subsequently, for each bond $\mathbf{r}_{i j}$ a vector of complex spherical harmonics $Y_{l}^{m}\left(\theta\left(\mathbf{r}_{i j}\right), \phi\left(\mathbf{r}_{i j}\right)\right)$ is calculated for a chosen $l$, with $m=-l, \ldots, l$, and $2 l+1$ parameters $Q_{l, m}(i)$ are obtained. These are defined as an average over bonds, i.e.,

$$
Q_{l, m}(i)=\frac{1}{N_{\mathcal{B}(i)}} \sum_{j \in \mathcal{B}(i)} Y_{l}^{m}\left(\theta\left(\mathbf{r}_{i j}\right), \phi\left(\mathbf{r}_{i j}\right)\right) .
$$


Finally, parameters $Q_{l}(i)$ and $\hat{W}_{l}(i)$ are obtained as

$$
Q_{l}(i)=\left(\frac{4 \pi}{2 l+1} \sum_{m=-l}^{l}\left|Q_{l, m}(i)\right|^{2}\right)^{1 / 2}
$$

and

$$
\hat{W}_{l}(i)=W_{l}(i) \times\left(\sum_{m=-l}^{l}\left|Q_{l, m}(i)\right|^{2}\right)^{3 / 2}
$$

where

$$
\begin{aligned}
W_{l}(i)=\sum_{\substack{m_{1}, m_{2}, m_{3} \\
m_{1}+m_{2}+m_{3}=0}} & \left(\begin{array}{ccc}
l & l & l \\
m_{1} & m_{2} & m_{3}
\end{array}\right) \\
& \times Q_{l, m_{1}}(i) Q_{l, m_{2}}(i) Q_{l, m_{3}}(i),
\end{aligned}
$$

and

$$
\left(\begin{array}{ccc}
l_{1} & l_{2} & l_{3} \\
m_{1} & m_{2} & m_{3}
\end{array}\right)
$$

is the Wigner $3 j$ symbol. Equipped with the values of $Q_{l}(i)$ and $\hat{W}_{l}(i)$ ordering can be studied by comparing them against reference values. Values calculated for common ideal structures are shown in Table 1.

\begin{tabular}{cccrr}
\hline Structure & \multicolumn{4}{c}{ Parameter } \\
& $Q_{4}$ & $Q_{6}$ & $\hat{W}_{4}$ & \multicolumn{1}{c}{$\hat{W}_{6}$} \\
\hline sc & 0.76376 & 0.35355 & 0.15932 & 0.01316 \\
bcc & 0.03637 & 0.51069 & 0.15932 & 0.01316 \\
fcc & 0.19094 & 0.57452 & -0.15932 & -0.01316 \\
hcp & 0.09722 & 0.48476 & 0.13410 & -0.01244 \\
icos & 0 & 0.66332 & 0 & -0.16975 \\
\hline
\end{tabular}

Table 1: Values of BOP parameters for typical ideal structures.

Bond-orientational order parameters are sensitive to symmetries. Using $Q_{l}$ and $\hat{W}_{l}$ it is possible to distinguish structures differing by the number of $l$-fold axes of symmetry. In practical applications parameters with $l=4$ and 6 are used most commonly, as they allow differentiating between most common packing types: sc, bcc, fcc and hcp. The BOP approach proves particularly useful for studying icosahedral ordering, as $\hat{W}_{6}$ is sensitive to structural features of icosahedra, a consequence of the fact that for a perfect icosahedron, characterized by five-fold symmetry, $\hat{W}_{6}$ assumes a negative value of -0.16975 , significantly different from values typical for cubic structures, which are close to zero (for sc and bcc $\hat{W}_{6}=0.01316$, for fcc $\hat{W}_{6}=$ $-0.01315)$. This advantage of $\hat{W}_{6}$ has been repeatedly exploited in studies of icosahedral ordering $[9,19$, 20, 22, 48, 54].

The BOP technique also runs into difficulties for liquid systems. Here they stem from the fact that BOPs are based on the concept of nearest neighbors. It has recently been shown [76] that the obtained picture of the short-range order in a liquid is sensitive to the operative definition of nearest neighbor, and the use of the original formulation due to Steinhardt et al. [4, 5] (using a distance-based cutoff $r_{\mathrm{c}}$ ) can overshadow the structural changes induced by the physics of a liquid system.

In Ref. [76] Mickel et al. not only highlighted the limitations of BOP, but also proposed a novel formulation, free from the arbitrariness of choosing a cutoff distance. This formulation invokes Voronoi analysis, 
defining common neighbors as two atoms sharing a face in a Voronoi diagram, leading to the following definition of an analog to $Q_{l}(i)$ :

$$
Q_{l}^{\prime}(i)=\left(\frac{4 \pi}{2 l+1} \sum_{m=-l}^{l}\left|Q_{l, m}^{\prime}(i)\right|^{2}\right)^{1 / 2}
$$

where

$$
Q_{l, m}^{\prime}(i)=\sum_{f \in \mathcal{V}(i)} \frac{a(i, f)}{A(i)} Y_{l}^{m}\left(\theta_{f}(i), \phi_{f}(i)\right) .
$$

In the above $\mathcal{V}(i)$ denotes the Voronoi polyhedron of particle $i$. The index $f$ runs over all faces of the polyhedron $\mathcal{V}(i)$. The surface area of face $f$ is denoted with $a(i, f)$, and $A(i)$ is the total surface area of the polyhedron $\mathcal{V}(i)$, i.e., $A(i)=\sum_{f \in \mathcal{V}(i)} a(i, f)$. Angles $\theta_{f}(i)$ and $\phi_{f}(i)$ are the spherical angles of the outer normal vector of facet $f$. The direction of this vector coincides with the bond vector $\mathbf{r}_{i j}$ that is used in conventional bond-orientational order analysis.

We will use the prime symbol appearing first in Eqs. (6)-(7) to distinguish BOPs obtained using Mickel's defintion from those obtained from Steinhardt's formulation (Eqs. (1)-(5)). While the expression for $Q_{l}^{\prime}(i)$ (Eq. (6)) is the same as that for $Q_{l}(i)$ (Eq. (2)), we note that $Q_{l, m}^{\prime}(i)$ (Eq. (7)) is defined considerably differently from $Q_{l, m}(i)$ (Eq. (1)). Not only does Mickel's formulation differ by the approach to defining the set of nearest neighbors (using Voronoi polyhedra and geometrical neighbors), but the contributions from each face $f$ (nearest neighbor $j$ ) are weighted by the ratio of the surface area of the face $a(i, f)$ to the total surface area of the polyhedron $A(i)$. This attenuates the contributions to $Q_{l, m}^{\prime}(i)$ due to spurious neighbors caused by thermal fluctuations, while not appreciably affecting $Q_{l}^{\prime}(i)$, making the approach considerably advantageous for studying liquid structure. An additional benefit of using a Voronoi-based definition of neighborhood is that the obtained parameters become continuous functions of atomic positions.

In Ref. [76] Mickel et al. did not define $\hat{W}_{l}^{\prime}$ which would correspond to Steinhardt's $\hat{W}_{l}$ (Eq. (3)). Following their line of reasoning, in this work we propose to define it as

$$
\hat{W}_{l}^{\prime}(i)=W_{l}^{\prime}(i) \times\left(\sum_{m=-l}^{l}\left|Q_{l, m}^{\prime}(i)\right|^{2}\right)^{3 / 2}
$$

where

$$
\begin{aligned}
W_{l}^{\prime}(i)=\sum_{\substack{m_{1}, m_{2}, m_{3} \\
m_{1}+m_{2}+m_{3}=0}} & \left(\begin{array}{ccc}
l & l & l \\
m_{1} & m_{2} & m_{3}
\end{array}\right) \\
& \times Q_{l, m_{1}}^{\prime}(i) Q_{l, m_{2}}^{\prime}(i) Q_{l, m_{3}}^{\prime}(i),
\end{aligned}
$$

and with the quantities $Q_{l, m}^{\prime}(i)$ given by Eq. (7). Similarly to $\hat{W}_{l}$, the parameters $\hat{W}_{l}^{\prime}$ remain rotationally invariant.

In the case of perfect sc, fcc, hcp and icos structures the values of parameters obtained using Mickel's formulation are identical to those obtained using the original formulation due to Steinhardt. This is a consequence of the fact that for each of these structures all faces of the Voronoi polyhedron have the same surface area. This is in contrast to the bcc structure, for which the values obtained using Mickel's formulation differ from those given in Table 1 , and assume instead the following values: $Q_{4}^{\prime}=0.22402, Q_{6}^{\prime}=0.56694$, $\hat{W}_{4}^{\prime}=-0.15932, \hat{W}_{6}^{\prime}=0.01316$.

In both formulations the parameter $\hat{W}_{6}^{\prime}$ assumes a highly negative value for the icosahedral structure, enabling the detection of icosahedral and icosahedron-like structures, which makes it highly useful for studying icosahedral ordering in liquids. 


\subsection{Novel approach}

In this work we present a novel approach to the structural analysis of disordered systems, geared towards liquid metal systems and identification of icosahedral ordering. The proposed approach is a hybrid of the Voronoi and BOP approaches, with local ordering in the vicinity of particle $i$ is described using a pair of descriptors: the Voronoi signature $\left(f_{3}(i), f_{4}(i), f_{5}(i), f_{6}(i)\right)$ and the $\hat{W}_{6}^{\prime}(i)$ parameter. The use of a Voronoi signature is aimed at extracting information on topological features of the nearest neighborhood. The use $\hat{W}_{6}^{\prime}$ augments analysis by assessing those features that are not captured in a Voronoi signature - it enables distinguishing polyhedra that have the same signature but differ with regard to shape. In particular $\hat{W}_{6}^{\prime}$ can be used to quantify the degree of icosahedrity of a particle's nearest neighborhood.

Since the proposed approach is aimed at liquid systems, following the determination of $\mathcal{V}(i)$ for each particle we proceed by eliminating small faces from the Voronoi polyhedra. We use a simple criterion, where a face $f$ is eliminated if its surface area $a(i, f)$ is below a given thershold, i.e.:

$$
\alpha(i, f)=\frac{a(i, f)}{\left(1 / N_{\mathcal{V}(i)}\right) A(i)}<\alpha_{\mathrm{sf}},
$$

where $N_{\mathcal{V}(i)}$ is the number of faces in polyhedron $\mathcal{V}(i)$. The above procedure eliminates faces whose area is below a fraction $\alpha_{\mathrm{sf}}$ of the mean face area in the original polyhedron $\mathcal{V}(i)$. Details on the choice of $\alpha_{\mathrm{sf}}$ will be given later.

The result of face elimination is a polyhedron $\mathcal{V}^{\prime}(i)$, with $N_{\mathcal{V}^{\prime}(i)}$ faces and a signature $\left(f_{3}^{\prime}(i), f_{4}^{\prime}(i), f_{5}^{\prime}(i), f_{6}^{\prime}(i)\right)$. We will use a convention where the prime symbol is used to distinguished "cleaned" polyhedra from the original polyhedra. Face elimination is only performed once for every particle $i$, i.e., $\mathcal{V}^{\prime}(i)$ does not undergo further clean up. We also note that the polyhedra are cleaned up independently - the fact that two particles $i$ and $j$ shared a face in the original diagram does not in general mean that they will share a face in the cleaned up diagram, for instance it may happen that the face due to $j$ will be removed from $\mathcal{V}(i)$, while the face due to $i$ will be retained in $\mathcal{V}(j)$. The goal here is filter the features that are not relevant to the topological analysis of a given polyhedron. The elimination can be seen as a map $\mathcal{V}(i) \rightarrow \mathcal{V}^{\prime}(i)$, employed only to facilitate the analysis of the signature $\left(f_{3}(i), f_{4}(i), f_{5}(i), f_{6}(i)\right)$ by mapping it to $\left(f_{3}^{\prime}(i), f_{4}^{\prime}(i), f_{5}^{\prime}(i), f_{6}^{\prime}(i)\right)$, whose signature is expected to be easier to interpret.

We stress that the above formulation is unique - the result of face elimination is independent on the order in which polyhedra, or faces within a given polyhedron, are processed. The criterion (10) is local only the ratio between the area of a face to the mean area of the polyhedron is considered.

The signature $\left(f_{3}^{\prime}(i), f_{4}^{\prime}(i), f_{5}^{\prime}(i), f_{6}^{\prime}(i)\right)$ is subsequently augmented with a descriptor of symmetries in the nearest neighborhood of the particle, i.e., the parameters $\hat{W}_{6}^{\prime}(i)$ determined based on $\mathcal{V}(i)$ and $a(i, f)$. We note that Eq. (7) uses contributions from $\mathcal{V}(i)$, and not the cleaned-up polyhedron $\mathcal{V}^{\prime}(i)$. This is because face elimination is only performed to clean up the structure in the space of Voronoi signatures. The use of $\mathcal{V}^{\prime}(i)$ would be counter-productive, as the parameters $\hat{W}_{6}^{\prime}(i)$ are not sensitive to the presence of small faces, and, furthermore, the use of $\mathcal{V}^{\prime}(i)$ would yield BOP parameters that would not be continuous in the atomic positions.

A naive implementation of such a double-pronged approach would be computationally expensive. The work of Stukowski [77], where commonly used approaches to structural analysis are compared with regard to their computational effort, assigns a cost of 100 to BOP, and 50 to Voronoi analysis, while simple approaches like the centrosymmetry parameter and CNA are assigned a cost of 1 and 3, respectively. In practice the cost can be severely mitigated through the use of of a highly-efficient library Voro++ [78] for Voronoi tesselation and recently proposed [79] fast simultaneous interpolation (FSI) to the determination of BOP. The latter technique can dramatically (up to 50-fold) reduce the effort associated with the determination of BOP [79]. 


\section{Results}

\subsection{Averaged structural features}

We begin the analysis with a comparison of averaged structural features. Fig. 1a shows the pair correlation functions $g_{2}(r)$ obtained from simulation, which we compare against results obtained through X-ray absorption spectroscopy postprocessed by reverse Monte Carlo technique (RMC-XAS) by Di Cicco et al. [9] and against DFT-MD results due to Ganesh et al. [22]. We find all three approaches to be in very good agreement for the first peak and to agree satisfactorily in the longer range.

Angular distribution functions $g_{3}(\theta)$ (Fig. 1b) also compare favorably between results obtained in this work and the results of Di Cicco et al. and Ganesh et al.. For each of the three temperatures the shapes of $g_{3}(\theta)$ curves are similar, with the positions and heights of the two main maxima and the minimum arount $90^{\circ}$ in good agreement.
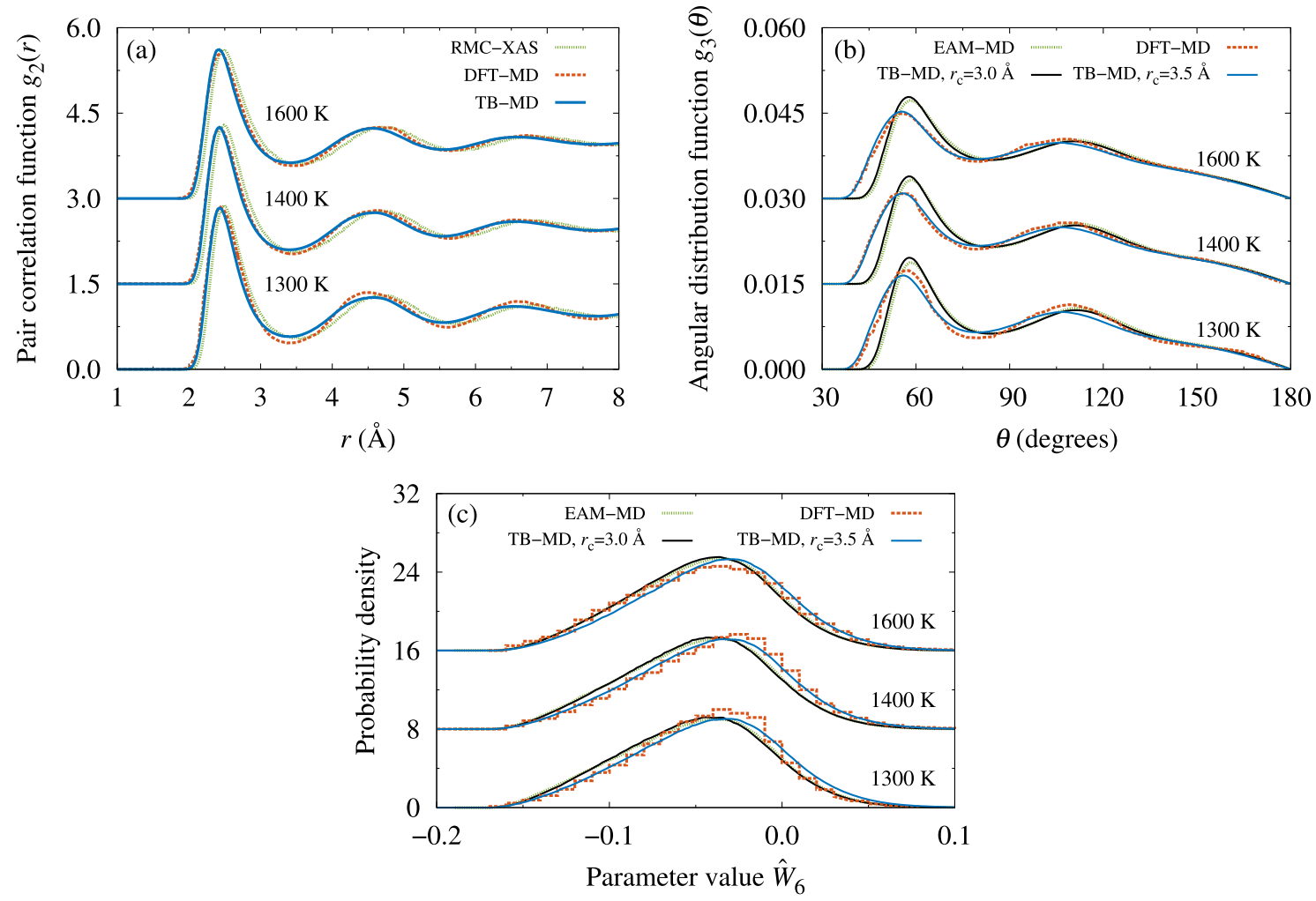

Figure 1: Pair correlation functions $g_{2}(r)$ (panel a), angular distribution functions $g_{3}(\theta)$ (panel b), and distributions of the parameter $\hat{W}_{6}$ (panel c) for temperatures of 1300, 1400 and $1600 \mathrm{~K}$. Curves labeled TB-MD correspond to this work, DFT-MD are the results of Ganesh et al. [22], RMC-XAS are due to Di Cicco et al. [9]. While Refs [22] and [9] studied liquid $\mathrm{Cu}$ at slightly different temperatures, those did not differ by more than $23 \mathrm{~K}$ from those used in this work. Curves labeled EAM-MD (panels b and c) are the results of Ref. [20] obtained from MD simulations using an embedded atom model (EAM) potential, but they can be interpreted as experimental, as Ref. [20] demonstrates their excellent agreement with RMC-XAS reults of Ref. [9]. TB-MD results in panels (b) and (c) are shown twice, once with a cut-off radius used by Celino et al. (3.0 $\AA$, black), and once with a cut-off radius used by Ganesh et al. (3.5 $\AA$, blue), and so the curves are meant to be compared in pairs (green vs. black and red vs. blue). The distributions of $\hat{W}_{6}$ in panel (c) used Steinhardt's definition, which is why a cut-off radius had to be chosen. 
The symmetries present in the structure (Fig. 1c) revealed by the distributions of $\hat{W}_{6}$ are also in good agreement between our results and those of Di Cicco et al. Crucially, the good agreement for low values of $\hat{W}_{6}$ indicates that the obtained structure is characterized by similar degree of icosahedral ordering. Quantitative comparison with the results of Ganesh et al. is made difficult by the stepwise character of results published in Ref. [22], but qualitative agreement is good.

The above demonstrates that NRL TB-MD yields structures whose short-range features (interatomic distances and angles, symmetries) agree very well with experimental results of Di Cicco et al. [9] and the results of Ganesh et al. [22] obtained at a more expensive level of theory (DFT-MD). The vastly lower computational effort of TB-MD enabled us to significantly increase the length- and time-scales of the simulation (by a factor of 5, and by more than 30-fold, respecitvely). This increase in the accessible time-scale in particular permits a better understanding of not only the structure, but its dynamics, as we will now demonstrate.

\subsection{Parameters of the analysis}

We began by calculating the descriptors $\left(f_{3}^{\prime}(i), f_{4}^{\prime}(i), f_{5}^{\prime}(i), f_{6}^{\prime}(i)\right)$ and $\hat{W}_{6}^{\prime}(i)$ for each particle in each time step, for each of the temperatures. Small faces were eliminated from the Voronoi polyhedra using a uniform value of $\alpha_{\mathrm{sf}}=0.075$, chosen based on numerical experiments, where the influence of the value of $\alpha_{\mathrm{sf}}$ on the obtained structure in the space of Voronoi signatures was ascertained.

Fig. 2 presents the distribution of $\alpha(i, f)$ for three temperatures and will serve to justify our choice. All three distributions are similar, with a bimodal character. The two peaks reflect the higher incidence of apparent (undesired) small faces and significantly larger faces of interest. The overlap of the two classes is, unfortunately, significant, and the fact that the intervening minimum does not reach zero implies that they cannot be separated perfectly. Moreover, a seemingly intuitive choice of the minimum around 0.5 as a cut-off would result in the elimination of a significant fraction of "valid" faces due to the long tail extending to the left of the second peak. We thus choose a conservative value of $\alpha_{\text {sf }}$ that is close to the first maximum in order to remove the worst offenders without obscuring the interesting features of the diagram. In the Appendix we present additional arguments supporting our choice of $\alpha_{\text {sf }}=0.075$, showing that it leads to the elimination of only undesired, excessively small faces.

Even such conservative elimination $\left(\alpha_{\mathrm{sf}}=0.075\right)$ greatly facilitates further analysis. For instance, for $T=$ $1300 \mathrm{~K}$ the raw Voronoi diagram comprises almost 3000 distinct polyhedra signatures $\left(f_{3}, f_{4}, f_{5}, f_{6}, f_{7}, \ldots\right)$, with only about $25 \%$ belonging to the easily-interpreted class $\left(0, f_{4}, f_{5}, f_{6}, 0, \ldots\right)$ (i.e., containing only tetra-, penta- and hexagonal faces). Following elimination this is reduced to only 1200 distinct signatures, with the ratio of easily-interpreted signatures as high as $60 \%$. This fraction is composed of almost 60 distinct polyhedra types, but only 17 of these occur with a frequency exceeding $1 \%$. It is these polyhedra - with easy to interpret signatures and significant occurence - that will be the focus of this work.

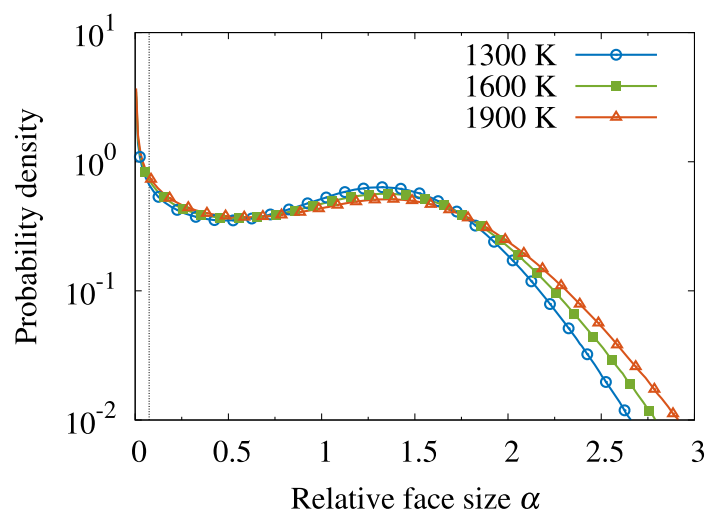

Figure 2: Distribution of face surface areas. The dashed vertical line indicates the chosen elimination cut-off $\alpha_{\text {sf }}=0.075$. 
In particular, we will concern ourselves with polyhedra with the following signatures: $(0,0,12,0)($ corresponding to icosahedra); $(1,0,9,3),(0,1,10,2)$ and $(0,2,8,2)$ (commonly accepted to be icosahedron-like); $(0,1,10,3),(0,2,8,1),(0,2,8,3)$ and $(0,2,8,4)$. Apart from the above 8 signatures, we will also focus on $(0,3,6,2),(0,3,6,3),(0,3,6,4)$ and $(0,3,6,5) ;(0,4,4,3),(0,4,4,4),(0,4,4,5)$ and $(0,4,4,6)$. As will be demonstrated later, these too constitute major structural motifs of liquid $\mathrm{Cu}$. All remaining polyhedra will be lumped together as "other polyhedra". While the above simplification (from over 1200 to only 16 signatures) may seem drastic, we point out that at $1300 \mathrm{~K}$ the above 16 signatures still account for over $50 \%$ of all observed polyhedra.

\subsection{Degree of icosahedrity}

We will now focus on the degree of icosahedrity of structural motifs found in liquid $\mathrm{Cu}$, using the parameter $\hat{W}_{6}^{\prime}$ as a main tool sensitive to icosahedral order.

Fig. 3 shows the distribution of $\hat{W}_{6}^{\prime}$ for $T=1300 \mathrm{~K}$ for a number of polyhedra types, focusing on polyhedra with 12 faces and 13-faced polyhedra with a signature $(0,1,10,2)$. Also shown is a distribution for "other polyhedra", and a total distribution serving as a baseline.

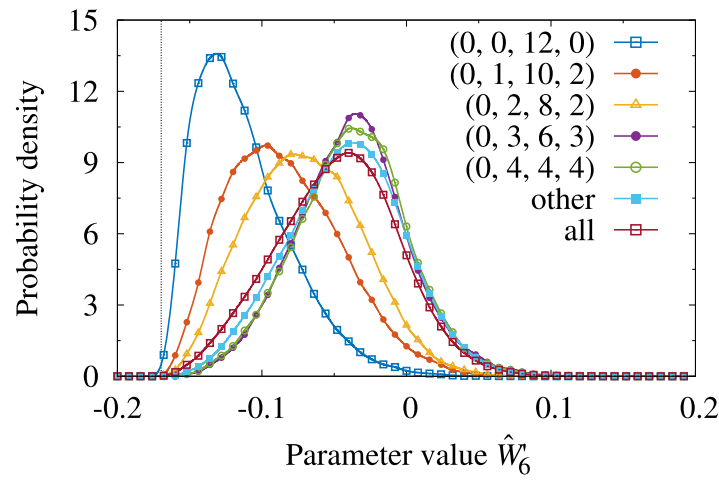

Figure 3: Distributions of $\hat{W}_{6}^{\prime}$ at $T=1300 \mathrm{~K}$ for selected types of polyhedra, "other polyhedra" and the total distribution. The dashed vertical line corresponds to $\hat{W}_{6}^{\prime}=-0.169754$ (perfect icosahedron).

Polyhedra with a signature of $(0,0,12,0)$ display highly negative values of $\hat{W}_{6}^{\prime}$, with a mean value of -0.109 , which is an indicator of a high degree of icosahedrity. Even with the long tail of the distribution (extending as far as beyond zero), its large positive skewness is a testament to the presence of marked fivefold orientational order in $(0,0,12,0)$ polyhedra. The situation is similar for $(0,1,10,2)$ polyhedra, which we recognize as highly icosahedron-like due to the low mean value of $\hat{W}_{6}^{\prime}(-0.080)$ and marked positive skewness.

In the case of $(0,2,8,2)$ polyhedra the positive skewness is less manifest; nevertheless negative values of $\hat{W}_{6}^{\prime}$ still dominate (mean value of -0.066 ), suggesting that these polyhedra are largely icosahedron-like, more so than $(0,3,6,3)$ and $(0,4,4,4)$ polyhedra for which mean $\hat{W}_{6}^{\prime}$ is -0.035 , and other polyhedra, with a mean of -0.038 . The mean over all polyhedra was -0.044 , indicating that the degree of icosahedrity of $(0,3,6,3)$ and $(0,4,4,4)$ polyhedra, and of other polyhedra is below the mean value. This is confirmed through visual inspection of the distributions - the total distribution is located to the left of the distributions corresponding to $(0,3,6,3),(0,4,4,4)$ and other polyhedra.

To extend the above analysis we will examine the statistical moments of $\hat{W}_{6}^{\prime}$ : its mean, $\mu\left(\hat{W}_{6}^{\prime}\right)$; standard deviation, $\sigma\left(\hat{W}_{6}^{\prime}\right)$; and Pearson's coefficient of skewness, $\gamma\left(\hat{W}_{6}^{\prime}\right)$. The values computed for all 16 types of polyhedra under consideration, together with values for other polyhedra and all polyhedra are shown in Fig. 4. Each polyhedron type is represented by a point on the $\mu\left(\hat{W}_{6}^{\prime}\right)-\gamma\left(\hat{W}_{6}^{\prime}\right)$ plane, and the standard deviation $\sigma\left(\hat{W}_{6}^{\prime}\right)$ is shown as an error bar on the horizontal axis. This representation facilitates the assessment of icosahedrity - points close to the top-left corner correspond to polyhedra with the largest degrees of 

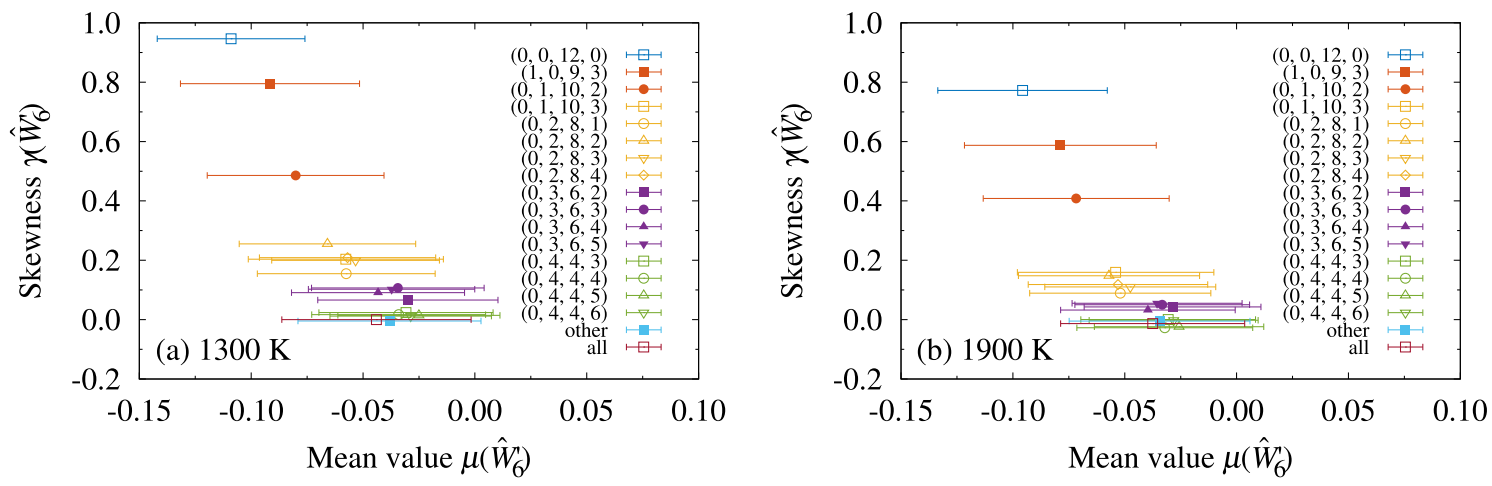

Figure 4: Degree of icosahedrity of considered polyhedra types. To facilitate analysis, polyhedra belonging to the same group (see text) were denoted by the same colors.

icosahedrity (highly negative $\mu\left(\hat{W}_{6}^{\prime}\right)$, large magnitude of $\gamma\left(\hat{W}_{6}^{\prime}\right)$ indicating large positive skewness), and conversely points located close to the bottom-right corner are characterized by a low degree of icosahedrity.

Fig. 4 makes it clear that highly icosahedral structures are $(0,0,12,0)$, followed by $(1,0,9,3)$ and $(0,1,10,2)$. A second, markedly distinct group of polyhedra that significantly resemble icosahedra are $(0,1,10,3),(0,2,8,1),(0,2,8,2),(0,2,8,3)$ and $(0,2,8,4)$. Polyhedra with lower degrees of icosahedrity are $(0,3,6,2),(0,3,6,3),(0,3,6,4),(0,3,6,5)$, and $(0,4,4,3),(0,4,4,4),(0,4,4,5),(0,4,4,6)$, and other polyhedra.

The above observations hold for the entire range of temperatures studied here, which can be inferred by comparing panels corresponding to the lowest and higher temperatures. While the increase in temperature results in a reduction in icosahedrity, the qualitative differences between groups of polyhedra remains even at $1900 \mathrm{~K}$.

To facilitate further discussion, we will now classify the polyhedra into six groups: $\mathcal{I}, \mathcal{J}, \mathcal{K}, \mathcal{L}, \mathcal{M}$ and $\mathcal{O}$, corresponding to groupings in the $\mu\left(\hat{W}_{6}^{\prime}\right)-\gamma\left(\hat{W}_{6}^{\prime}\right)$ plane. The group $\mathcal{I}$ will be composed solely of $(0,0,12,0)$ polyhedra, whose icosahedrity is highest. The group $\mathcal{J}$ will encompass highly-icosahedron-like polyhedra $(1,0,9,3)$ and $(0,1,10,2)$. Moderately icosahedron-like polyhedra $(0,1,10,3),(0,2,8,1),(0,2,8,2)$, $(0,2,8,3)$ and $(0,2,8,4)$ will be classified as $\mathcal{K}$. Polyhedra that are not measurably icosahedron-like, i.e., are characterized by a degree of icosahedrity that is below mean or comparable to mean will be classified as $\mathcal{L}((0,3,6,2),(0,3,6,2),(0,3,6,4)$ and $(0,3,6,5))$ or $\mathcal{M}((0,4,4,3),(0,4,4,4),(0,4,4,5)$ and $(0,4,4,6))$. The final group $\mathcal{O}$ will encompass other polyhedra. The degree of icosahedrity is not the sole criterion for the above classification - we note in passing that each group comprises polyhedra with similar signatures, typically differing only with regard to the number of hexagonal faces.

The above analysis based on the $\hat{W}_{6}^{\prime}$ parameter markedly facilitates structural interpretation, but is limited by its static nature that cannot reveal the dynamics of structural transformations taking place in liquid $\mathrm{Cu}$ in time or with changes in temperature. We will elucidate these in the text that follows.

\subsection{Frequencies of occurrence}

We begin by examining how the structure is affected by temperature. Fig. 5 presents the histograms for the frequencies $n$ with which the 16 considered types of polyhedra occur, for three temperatures: $1300 \mathrm{~K}$, $1600 \mathrm{~K}$ and $1900 \mathrm{~K}$. Except for signatures $(0,3,6,2)$ and $(0,4,4,3)$, for all polyhedra types the frequency with which they occur increases with a decrease in temperature. This effect is most pronounced for $(0,0,12,0)$ polyhedra, which occur more frequently by a factor of $n(1300 \mathrm{~K}) / n(1900 \mathrm{~K})=2.5$ at the lowest temperature.

A similar, but slightly less pronounced effect is observed for the most icosahedron-like signatures - the same ratio is $\approx 2$ for $(1,0,9,3)$ and $\approx 1.8$ for $(0,1,10,2)$. Similarly, a large value is obtained for $(0,1,10,3)$ polyhedra $(n(1300 \mathrm{~K}) / n(1900 \mathrm{~K})=1.9)$. For the remaining polyhedra in $\mathcal{K}$ (with the sole exception of $(0,2,8,1))$ we obtained values of $n(1300 \mathrm{~K}) / n(1900 \mathrm{~K})$ in the range $1.5-1.7$. 


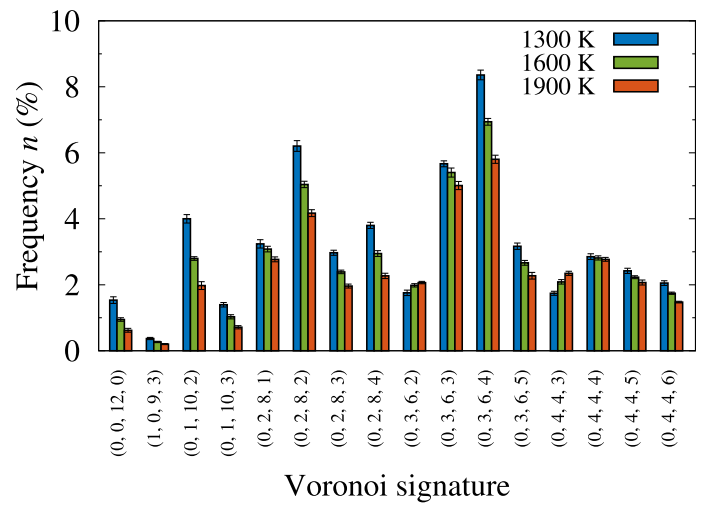

Figure 5: Frequencies of occurrence of each Voronoi polyhedra.

The above indicates that at temperatures close to the melting temperature icosahedral ordering is more strongly favored, as for the remaining polyhedra the relative increases in the frequencies of occurrence were much smaller, typically below 1.1 (two exceptions were $(0,3,6,4)$ and $(0,3,6,5)$, for which $n(1300 \mathrm{~K}) / n(1900 \mathrm{~K}) \approx 1.4)$. At $1300 \mathrm{~K}$ polyhedra with large degrees of icosahedrity constitute almost $24 \%$ of all polyhedra, with $\mathcal{K}$ being the most abudant $(17.6 \%$ of total), followed by $\mathcal{J}$ ( $4.3 \%$ of total).

In Fig. 6 we plot five representative dependencies $n(T)$. We find each of these to be well-represented by the Arrhenius equation

$$
n(T)=n_{\infty} \exp \left(-\frac{E_{\mathrm{f}}}{k_{\mathrm{B}} T}\right)
$$

corresponding to straight lines in Fig. 6. The relevant parameters are given in Table 2 .

Of particular interest is the parameter $E_{\mathrm{f}}$, which can be understood as a mean energy of formation of a given structural motif surrounded by a thermodynamically average embedding. Negative values correspond to increased stability of a particular motif.

Fig. 7 shows the correlation between $E_{\mathrm{f}}$ and $\mu\left(\hat{W}_{6}^{\prime}\right)$ (for $1300 \mathrm{~K}$ ). The two parameters are seen to correlate strongly - structures with highly negative $\hat{W}_{6}^{\prime}$ have the lowest (most negative) energies of formation, down to $-0.3 \mathrm{eV}$ for icosahedra $(0,0,12,0)$ and -0.25 and $-0.2 \mathrm{eV}$ for icosahedron-like signatures $(0,1,10,2)$ and $(1,0,9,3)$. Further on, for moderately icosahedron-like polyhedra in $\mathcal{K}$ the energies of formation are between -0.2 and $-0.05 \mathrm{eV}$, and as such are lower than the reference value of $E_{\mathrm{f}}=0$ corresponding to the thermodynamically mean structural motif. Interestingly, even some polyhedra from $\mathcal{L}$ and $\mathcal{M}$ can achieve negative energies of formation, but with modest magnitudes, typically not exceeding $-0.1 \mathrm{eV}$. The remaining polyhedra $(0,4,4,3)$ and $(0,3,6,2)$ are characterized by positive energies of formation, similarly to other polyhedra $(\mathcal{O})$, which are also characterized by low stability.

The energy of formation $E_{\mathrm{f}}$ enables the assessment of stability of given structures, but it does not provide an exhaustive description of the energetics of liquid $\mathrm{Cu}$, for example it does not yield information on the heights of energy barriers associated with the structures. This can be remediated by analyzing the dynamics of structural transitions, which we will proceed to do now. 


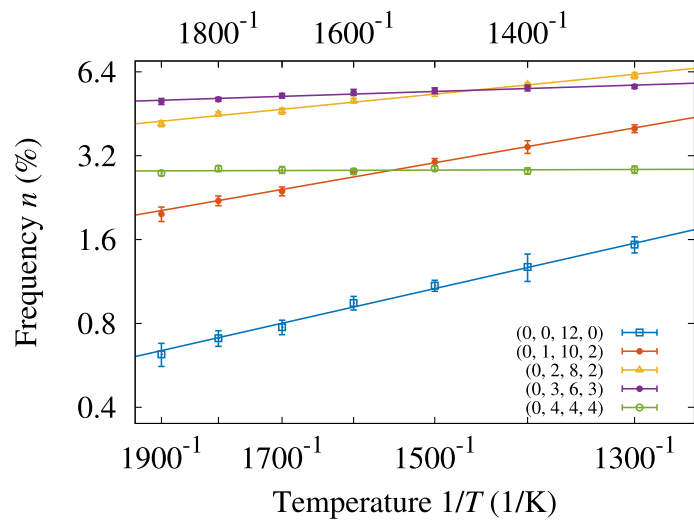

Figure 6: Dependence of the frequency of occurrence $n$ on temperature $T$ for selected Voronoi signatures. Straight lines are fit to the Arrhenius equation (see text).

\begin{tabular}{cccccc}
\hline Group & Voronoi signature & \multicolumn{2}{c}{ Frequency $n(\%)$} & \multicolumn{2}{c}{ Temp. dependence } \\
& $\left(f_{3}^{\prime}, f_{4}^{\prime}, f_{5}^{\prime}, f_{6}^{\prime}\right)$ & $1300 \mathrm{~K}$ & $1900 \mathrm{~K}$ & $E_{\mathrm{f}}(\mathrm{eV})$ & $n_{\infty}(\%)$ \\
\hline $\mathcal{I}$ & $(0,0,12,0)$ & 1.53 & 0.62 & -0.315 & 0.09 \\
\hline $\mathcal{J}$ & $(1,0,9,3)$ & 0.37 & 0.20 & -0.196 & 0.06 \\
& $(0,1,10,2)$ & 4.00 & 1.98 & -0.242 & 0.46 \\
\hline $\mathcal{K}$ & $(0,1,10,3)$ & 1.39 & 0.71 & -0.227 & 0.18 \\
& $(0,2,8,1)$ & 3.24 & 2.77 & -0.054 & 2.04 \\
& $(0,2,8,2)$ & 6.21 & 4.17 & -0.137 & 1.84 \\
& $(0,2,8,3)$ & 2.97 & 1.96 & -0.137 & 0.88 \\
& $(0,2,8,4)$ & 3.80 & 2.27 & -0.174 & 0.81 \\
\hline $\mathcal{L}$ & $(0,3,6,2)$ & 1.76 & 2.07 & 0.055 & 2.94 \\
& $(0,3,6,3)$ & 5.67 & 5.01 & -0.044 & 3.86 \\
& $(0,3,6,4)$ & 8.36 & 5.80 & -0.125 & 2.76 \\
& $(0,3,6,5)$ & 3.17 & 2.27 & -0.119 & 1.10 \\
\hline $\mathcal{M}$ & $(0,4,4,3)$ & 1.74 & 2.35 & 0.101 & 4.37 \\
& $(0,4,4,4)$ & 2.85 & 2.77 & -0.004 & 2.75 \\
& $(0,4,4,5)$ & 2.42 & 2.07 & -0.056 & 1.47 \\
$\mathcal{A}$ & $(0,4,4,6)$ & 2.05 & 1.47 & -0.106 & 0.80 \\
\hline $\mathcal{O}$ & other & 48.5 & 61.5 & 0.083 & 102 \\
\hline
\end{tabular}

Table 2: Frequencies of occurrence of considered polyhedra signatures, and the parameters for their dependence on temperature. 
Figure 7: Correlation between $E_{\mathrm{f}}$ and $\mu\left(\hat{W}_{6}^{\prime}\right)$. Dashed lines are meant as a guide for the eye, corresponding to the mean degree of icosahedrity and formation energies for all polyhedra (maroon) and other polyhedra (light blue).

\subsection{Lifetimes}

Due to significant thermal activation, the short-range order in a liquid undergoes continual transitions. Characterizing the dynamics of these transitions (identifying them and quantifying their rates) is made difficult by the large variety of structural motifs in a liquid, and the significant uncertainties caused by thermal motions.

The approach we propose below facilitates the analysis of structural transitions in liquid systems. This is due to the marked simplification of the Voronoi signature space following face elimination, and to restricting the analysis to transitions between most common structural motifs $(16+1$ polyhedra signatures $)$ with well-defined characteristics $(5+1$ polyhedra groups $\mathcal{I}-\mathcal{O})$.

We begin by introducing basic concepts. We will say that a given particle $i$ underwent a transition at time $t+h / 2$ if any of the indices $f_{e}^{\prime}(i)$ describing its Voronoi polyhedron changed between $t$ and $t+h$, i.e., $f_{e}^{\prime}(i, t) \neq f_{e}^{\prime}(i, t+h)$ for any $e \geq 3$. Crucially, we will also posit that particle $i$ underwent a transition at $t+h / 2$ if its set of nearest neighbors changed between $t$ and $t+h$. By nearest neighbors of $i$ we denote the set of particles that, following face elimination, contribute a face to $\mathcal{V}^{\prime}(i)$. Under this definition it is possible for a signature of $i$ to undergo a transtion into itself. We will say that a lifetime of a polyhedron was $t_{\text {life }}$, starting at $t$ if it underwent transitions at $t-h / 2$ (birth) and $t+t_{\text {life }}+h / 2$ (death), and it did not undergo a transition between these instants. In the definitions above $h$ is the MD timestep used in the simulations. The temporal discretization introduces an uncertainty of $h / 2$ into the determination of a polyhedron's time of birth and death, and, consequently, an uncertainty of $h$ into its determined lifetime. Since the timestep used was small ( $h=\Delta t=0.5 \mathrm{fs}$ ), this uncertainty does not hamper further analysis.

By examining the trajectories of particles in the space of Voronoi signatures $\left(f_{3}^{\prime}(i), f_{4}^{\prime}(i), f_{5}^{\prime}(i), f_{6}^{\prime}(i)\right)$ we can easily determine the instants when transitions occured, and subsequently the lifetimes of all polyhedra types. By averaging these over particles and time we can establish the distributions of lifetimes $P_{\text {life }}\left(t ;\left(f_{3}^{\prime}, f_{4}^{\prime}, f_{5}^{\prime}, f_{6}^{\prime}\right)\right)$. This survival function describes the probability that a polyhedron with a Voronoi signature $\left(f_{3}^{\prime}, f_{4}^{\prime}, f_{5}^{\prime}, f_{6}^{\prime}\right)$ will live for at least $t$.

Fig. 8 shows the survival functions $P_{\text {life }}\left(t ;\left(f_{3}^{\prime}, f_{4}^{\prime}, f_{5}^{\prime}, f_{6}^{\prime}\right)\right)$ of representative signatures $(0,0,12,0)$, $(0,1,10,2),(0,2,8,2),(0,3,6,3),(0,4,4,4)$ and other polyhedra at $T=1300 \mathrm{~K}$. For reference, the survival function of all polyhedra is also shown. Over 850,000 of full lifetimes were observed, with lifetimes of $(0,0,12,0)$ polyhedra (relatively uncommon) sampled with 5500 observations, indicating a good degree of sampling. 


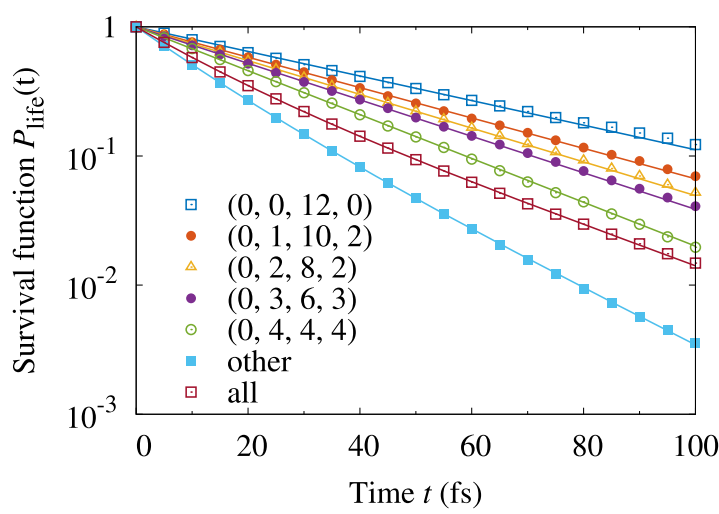

Figure 8: Survival functions for $T=1300 \mathrm{~K}$. Solid lines represent fits (12) and (13) (see text).

In all but two cases, the examined distribution $P_{\text {life }}(t)$ were well described by the functional form

$$
P_{\text {life }}(t)=\exp \left(-\frac{t}{\tau}\right)
$$

with one free parameter $\tau$, representing a mean lifetime $\left\langle t_{\text {life }}\right\rangle$. The two cases that could not be well fitted with this form were other polyhedra and all polyhedra, whose behavior did not follow a purely exponential character. We attribute this to the large structural variety of these two groups. For other polyhedra and all polyhedra a good fit was obtained with the functional form

$$
P_{\text {life }}(t)=a \exp \left(-\frac{t}{\tau_{1}}\right)+(1-a) \exp \left(-\frac{t}{\tau_{2}}\right)
$$

with three free parameters: $a, \tau_{1}$ and $\tau_{2}$, and a mean lifetime $\left\langle t_{\text {life }}\right\rangle=a \tau_{1}+(1-a) \tau_{2}$. The obtained fits are shown as solid lines in Fig. 8.

Fig. 9 shows how the mean lifetime $\left\langle t_{\text {life }}\right\rangle$ depends on temperature for a number of selected signatures. In all cases the mean lifetime decreases with an increase in temperature. Further analysis revealed that the dependence $\left\langle t_{\text {life }}\right\rangle(T)$ is well-described by the Arrhenius relation

$$
\left\langle t_{\text {life }}\right\rangle(T)=t_{\infty} \exp \left(-\frac{E_{\mathrm{a}}}{k_{\mathrm{B}} T}\right) .
$$

The activation energy $E_{\mathrm{a}}$ can be interpreted as the mean thermal energy required for a given structural motif to transform into a different motif (or itself), having overcome local potential barriers.

The obtained Arrhenius fits are shown in solid lines in Fig. 9 and are seen to be very good fits to the data. Table 3 summarizes the values of $t_{\infty}$ and $E_{a}$, and the mean lifetimes $\left\langle t_{\text {life }}\right\rangle$ for the lowest and highest temperatures studied for all signatures under study.

The results shown above indicate that icosahedra $(\mathcal{I},(0,0,12,0))$ are characterized by the longest lifetimes, living on average for $30 \mathrm{fs}($ at $1900 \mathrm{~K}$ ) to $45 \mathrm{fs}$ (at $1300 \mathrm{~K}$ ). These lifetimes are, respectively, 2.5$(1900 \mathrm{~K})$ and 2.9 -fold $(1300 \mathrm{~K})$ longer than the lifetimes of other polyhedra $(\mathcal{O})$, whose lifetimes are the shortest of all groups. Similarly, a comparison with the mean picture (i.e., against all polyhedra) indicates that icosahedra are the most stable motifs in liquid $\mathrm{Cu}$ (lifetimes longer by a factor of 2.1 (1900 K) to $2.2(1300 \mathrm{~K}))$. The second-longest lifetimes $\left\langle t_{\text {life }}\right\rangle$ are those of $(0,2,8,1)$, which on average last for $37 \mathrm{fs}$ at $1300 \mathrm{~K}$, with the lifetimes of $(0,1,10,2)$ being similar. 


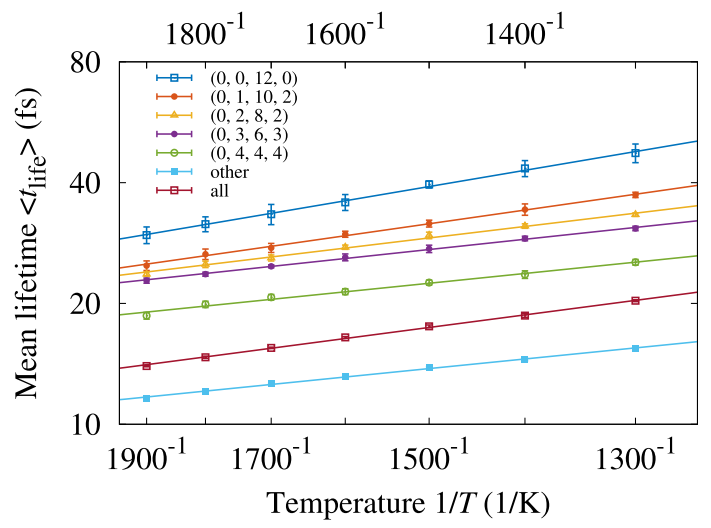

Figure 9: Dependence of mean lifetime on temperature. Solid lines denote fits to (14) (see text).

\begin{tabular}{cccccr}
\hline Group & $\begin{array}{c}\text { Voronoi signature } \\
\left(f_{3}^{\prime}, f_{4}^{\prime}, f_{5}^{\prime}, f_{6}^{\prime}\right)\end{array}$ & \multicolumn{2}{c}{ Lifetime $\left\langle t_{\text {life }}\right\rangle(\mathrm{fs})$} & \multicolumn{2}{c}{ Temp. dependence } \\
& $(0,0,12,0)$ & 45.6 & 29.7 & 0.169 & 10.60 \\
\hline $\mathcal{I}$ & $(1,0,9,3)$ & 19.5 & 15.0 & 0.089 & 8.86 \\
$\mathcal{J}$ & $(0,1,10,2)$ & 36.9 & 25.0 & 0.142 & 10.53 \\
\hline $\mathcal{K}$ & $(0,1,10,3)$ & 27.9 & 19.8 & 0.125 & 9.18 \\
& $(0,2,8,1)$ & 37.4 & 27.5 & 0.111 & 14.11 \\
& $(0,2,8,2)$ & 33.2 & 23.9 & 0.120 & 11.52 \\
& $(0,2,8,3)$ & 27.1 & 19.5 & 0.112 & 9.96 \\
& $(0,2,8,4)$ & 26.7 & 19.1 & 0.120 & 9.14 \\
\hline $\mathcal{L}$ & $(0,3,6,2)$ & 29.2 & 22.7 & 0.120 & 11.52 \\
& $(0,3,6,3)$ & 30.7 & 22.9 & 0.106 & 11.95 \\
& $(0,3,6,4)$ & 28.7 & 20.4 & 0.123 & 9.59 \\
& $(0,3,6,5)$ & 23.5 & 17.1 & 0.116 & 8.28 \\
\hline $\mathcal{M}$ & $(0,4,4,3)$ & 27.4 & 21.5 & 0.086 & 12.61 \\
& $(0,4,4,4)$ & 25.6 & 18.8 & 0.101 & 10.23 \\
& $(0,4,4,5)$ & 23.1 & 16.8 & 0.113 & 8.33 \\
$\mathcal{A}$ & $(0,4,4,6)$ & 21.9 & 15.8 & 0.114 & 7.89 \\
\hline $\mathcal{O}$ & other & 15.6 & 11.8 & 0.100 & 6.35 \\
\hline \multirow{2}{*}{ ath } & all & 20.4 & 14.1 & 0.131 & 6.33 \\
\hline & & & & &
\end{tabular}

Table 3: Mean lifetimes for all polyhedra types.

Among all considered polyhedra (groups $\mathcal{I}-\mathcal{M})$, only $(1,0,9,3)$ polyhedra have lifetimes that are shorter than the reference value obtained by averaging over all polyhedra. This strongly suggests that these polyhedra correspond to a structural motif that arises as a consequence of a mild thermal fluctuation, and the energy barriers that separate it from nearby structural motifs are low. This argument is made stronger by the low activation energy $E_{\mathrm{a}}$, which for $(1,0,9,3)$ polyhedra was only $0.089 \mathrm{eV}$, the lowest of all studied types of polyhedra. This leads us to presume that $(0,1,9,3)$ polyhedra can transform into icosahedra by expending minimal amounts of energy, with additional corroboration coming from the close vicinity between the two signatures - $(0,0,12,0)$ differs only by an introduction of a single triangular face at the vertex where 
three faces of the icosahedron meet. The above is in line with the earlier analysis of $\hat{W}_{6}^{\prime}$, which demonstrated that $(1,0,9,3)$ polyhedra are closest to icosahedra with regard to orientational order, and can be reasonably seen as a practically-icosahedral structural motif.

Our results are in line with those of Hao et al. [49] for metallic liquid $\mathrm{Zr}_{35} \mathrm{Cu}_{65}$, who, for $T=1300 \mathrm{~K}$, obtained a mean polyhedron lifetime of $27 \mathrm{fs}$ (compared to $20.4 \mathrm{fs}$ we obtained for liquid $\mathrm{Cu}$ ). Furthermore, Ref. [49] also shows that $(0,0,12,0)$ polyhedra are characterized by longest lifetimes, about 3 times longer than the average over all polyhedra (compared to 2.2 we obtained for liquid $\mathrm{Cu}$ ), again for $T=1300 \mathrm{~K}$. Finally, Ref. [49] reports that $(0,1,10,2)$ and $(0,2,8,2)$ polyhedra display longer lifetimes, which we also observe in the case of liquid $\mathrm{Cu}$.

\subsection{Structural transitions}

The lifetime analysis carried out above demonstrates that icosahedron-like structures persist for longer in the liquid, and do not transform readily. We will now augment this analysis by examining which of the possible structural transitions are actually realized and at what rates.

Quantitative analysis of structural transitions is an arduous task mostly due to the large number of possible transitions - examining $16+1$ types of polyhedra necessitates examining as many as $17 \times 17=289$ possible transitions. In the interest of clarity we will instead focus on transitions between the groups $\mathcal{I}-\mathcal{M}$ and $\mathcal{O}$ distinguished earlier, which reduces the number of possible transitions to $6 \times 6=36$.

We will now introduce necessary concepts. By $\mathcal{X} \rightarrow \mathcal{Y}$ we will denote a transition whereby a polyhedron from $\mathcal{X}$ transforms into a polyhedron from $\mathcal{Y}$, where $\mathcal{X}$ and $\mathcal{Y}$ can take any of the values $\mathcal{I}, \mathcal{J}, \mathcal{K}, \mathcal{L}, \mathcal{M}$ and $\mathcal{O}$. This is equivalent to a death of a polyhedron from $\mathcal{X}$ and a birth of a polyhedron from $\mathcal{Y}$. Under this notation, a transition of $(0,0,12,0)$ into $(0,1,10,2)$ will be denoted as $\mathcal{I} \rightarrow \mathcal{J}$, a transition of $(0,0,12,0)$ into $(0,2,8,2)$ will be denoted as $\mathcal{I} \rightarrow \mathcal{K}$, and an intra-group transition of $(0,2,8,1)$ into $(0,2,8,2)$ will be denoted as $\mathcal{K} \rightarrow \mathcal{K}$. In accordance with the operational definition of a transition, it is possible to observe an intra-group transformation that proceeds without a change in the Voronoi signature, resulting instead from a change in the set of neighbors.

To facilitate presentation, by $\mathcal{X} \rightarrow \mathcal{A}$ we will denote a transition where a polyhedron from $\mathcal{X}$ transforms into a polyhedron from any group $\mathcal{A}$. Thus, $\mathcal{A} \rightarrow \mathcal{A}$ will denote any transition. The number of observed

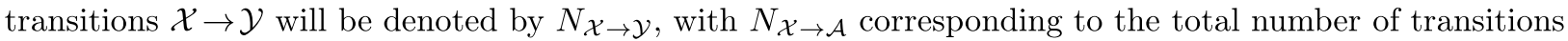
of polyhedra from $\mathcal{X}$, and $N_{\mathcal{A} \rightarrow \mathcal{A}}$ corresponding to the total number of observed transitions. We will use the notation $\mathcal{X} \rightleftharpoons \mathcal{Y}$ to denote both transitions between $\mathcal{X}$ and $\mathcal{Y}$, i.e., $\mathcal{X} \rightarrow \mathcal{Y}$ and $\mathcal{Y} \rightarrow \mathcal{X}$.

Given the quantities $N_{\mathcal{X} \rightarrow \mathcal{Y}}, N_{\mathcal{X} \rightarrow \mathcal{A}}$ and $N_{\mathcal{A} \rightarrow \mathcal{A}}$, one way to quantify the relative incidence of transitions $\mathcal{X} \rightarrow \mathcal{Y}$ is through

$$
F_{\mathcal{X} \rightarrow \mathcal{Y}}=\frac{N_{\mathcal{X} \rightarrow \mathcal{Y}}}{N_{\mathcal{A} \rightarrow \mathcal{A}}}
$$

which we will term the transition frequency of $\mathcal{X} \rightarrow \mathcal{Y}$. This quantitiy yields the fraction of all transitions constituted by $\mathcal{X} \rightarrow \mathcal{Y}$, and informs on the relevance of the transition $\mathcal{X} \rightarrow \mathcal{Y}$ in the grand picture. A comparison between $F_{\mathcal{X} \rightarrow \mathcal{Y}}$ and $F_{\mathcal{Y} \rightarrow \mathcal{X}}$ will yield valuable information, with large differences indicating significant asymmetries in the transition dynamics, highlighting paths along which transitions proceed, as shown in the analysis that follows.

Another measure that can yield insight into the incidence of transitions is

$$
T_{\mathcal{X} \rightarrow \mathcal{Y}}=\frac{N_{\mathcal{X} \rightarrow \mathcal{Y}}}{N_{\mathcal{X} \rightarrow \mathcal{A}}} \times \frac{1}{n(\mathcal{Y})}
$$

Here, the ratio $N_{\mathcal{X} \rightarrow \mathcal{Y}} / N_{\mathcal{X} \rightarrow \mathcal{A}}$ informs on the fraction of transitions from $\mathcal{X}$ realized by transitions into $\mathcal{Y}$. The factor $n(\mathcal{Y})^{-1}$, which denotes the frequency of occurrence of polyhedra from $\mathcal{Y}$, allows relating $N_{\mathcal{X} \rightarrow \mathcal{Y}} / N_{\mathcal{X} \rightarrow \mathcal{A}}$ to the expected frequency of $\mathcal{X} \rightarrow \mathcal{Y}$ transformations given by $n(\mathcal{Y})$, i.e., to the frequency that would be observed if all transformations were equally probable, with polyhedra from $\mathcal{X}$ transforming equally often into polyhedra from all groups ${ }^{1}$.

\footnotetext{
${ }^{1}$ Strictly speaking, $n(\mathcal{Y})^{-1}$ in eq. (16) should be replaced by $(1-1 / N) / n(\mathcal{Y})$ (if $\left.\mathcal{X} \neq \mathcal{Y}\right)$ or $(1-1 / N) /(n(\mathcal{Y})-1 / N)($ if $\mathcal{X}=\mathcal{Y})$. However, with a large number of particles $N$, the factor $1 / N$ can be neglected, yielding $n(\mathcal{Y})^{-1}$.
} 


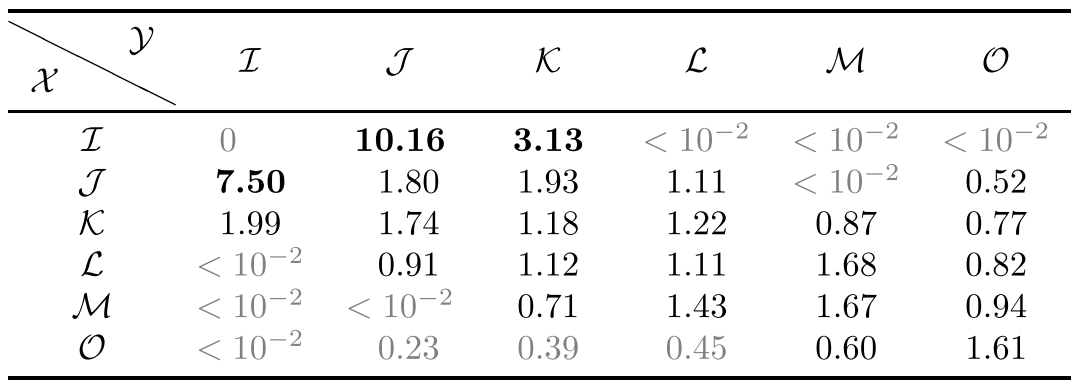

Table 4: Values of $T_{\mathcal{X} \rightarrow \mathcal{Y}}$ for $T=1300 \mathrm{~K}$.

The measure $T_{\mathcal{X} \rightarrow \mathcal{Y}}$, which we will term the transition tendency, can offer additional insight. For instance $T_{\mathcal{X} \rightarrow \mathcal{Y}} \gg 1$ would mean that transitions $\mathcal{X} \rightarrow \mathcal{Y}$ are realized much more often compared to a static, mean structural picture, corresponding to polyhedra from $\mathcal{X}$ readily transforming into polyhedra from $\mathcal{Y}$. Observing a large value for $T_{\mathcal{X} \rightarrow \mathcal{Y}}$ can indicate significant structural similarity between polyhedra from $\mathcal{X}$ and those from $\mathcal{Y}$. Conversely, $T_{\mathcal{X} \rightarrow \mathcal{Y}} \ll 1$ indicates that transitions $\mathcal{X} \rightarrow \mathcal{Y}$ do not happen readily, and polyhedra in $\mathcal{X}$ and $\mathcal{Y}$ are not structurally similar.

The fact that $T_{\mathcal{X} \rightarrow \mathcal{Y}}$ and $T_{\mathcal{Y} \rightarrow \mathcal{X}}$ are, in general, not equal is apparent from the definition. A comparison between $T_{\mathcal{X} \rightarrow \mathcal{Y}}$ and $T_{\mathcal{Y} \rightarrow \mathcal{X}}$ can indicate asymmetries in the dynamical picture of transitions.

In Table 4 and 5, we report the values of $T_{\mathcal{X} \rightarrow \mathcal{Y}}$ and $F_{\mathcal{X} \rightarrow \mathcal{Y}}$ for $T=1300 \mathrm{~K}$. We begin by examining the transition tendencies $T_{\mathcal{X}} \rightarrow \mathcal{Y}$. In the interest of clarity, in Table 4 we highlighted particulary common transitions $\left(T_{\mathcal{X} \rightarrow \mathcal{Y}}>2\right)$ in bold, and greyed out rare transitions $\left(T_{\mathcal{X}} \rightarrow \mathcal{Y}<0.5\right)$. Transitions $\mathcal{I} \rightarrow J$ and $\mathcal{J} \rightarrow \mathcal{I}$, occur the most readily, i.e., they are more common by a factor of $T_{\mathcal{I} \rightarrow \mathcal{J}}=10.2$ and $T_{\mathcal{J} \rightarrow \mathcal{I}}=7.5$ than would be indicated by the mean structural picture. This hints that polyhedra $(0,0,12,0)$ are structurally similar to $(1,0,9,3)$ and $(0,1,10,2)$. Relatively large values of $T_{\mathcal{X}} \rightarrow \mathcal{Y}$ were also observed for transitions $\mathcal{I} \rightarrow \mathcal{K}(3.1), \mathcal{J} \rightarrow \mathcal{K}(1.9)$ and transitions $\mathcal{K} \rightarrow \mathcal{I}$ and $\mathcal{K} \rightarrow \mathcal{J}$ (values of $1.7-1.9$ ). This indicates that polyhedra from $\mathcal{K}$ are also structurally similar to icosahedra.

The above results suggest that polyhedra in group $\mathcal{K}$ constitute intermediate structural motifs between structures with clear fivefold orientational order (grups $\mathcal{I}$ and $\mathcal{J}$ ), and other structural motifs (groups $\mathcal{L}$, $\mathcal{M}$ and $\mathcal{O}$ ). The intermediate nature of structures in $\mathcal{K}$ is further corroborated by the moderately large values of $T_{\mathcal{K} \rightarrow \mathcal{A}}$, viz. $\quad T_{\mathcal{K} \rightarrow \mathcal{L}}=1.2, T_{\mathcal{K} \rightarrow \mathcal{M}}=0.87, T_{\mathcal{K} \rightarrow \mathcal{O}}=0.77$. The situation is somewhat similar for polyhedra in group $\mathcal{L}$, which readily transform into polyhedra from $\mathcal{J}, \mathcal{K}, \mathcal{M}$ and $\mathcal{O}$. However, in this case practically no transitions to and from $\mathcal{I}$ are observed - values of $T_{\mathcal{I} \rightarrow \mathcal{L}}$ and $T_{\mathcal{L} \rightarrow \mathcal{I}}$ are in the order of $10^{-3}$. This indicates that polyhedra in $\mathcal{L}$ are not icosahedron-like. Similarly infrequent (values of the order of $10^{-3}$ ) are transitions $\mathcal{I} \rightarrow \mathcal{M}, \mathcal{M} \rightarrow \mathcal{I}$ and $\mathcal{J} \rightarrow \mathcal{M}, \mathcal{M} \rightarrow \mathcal{J}$, suggesting that polyhedra in group $\mathcal{M}$ are structurally very far from icosahedra. Similarly low transition tendencies were recorded for $\mathcal{O} \rightarrow \mathcal{I}$ and $\mathcal{I} \rightarrow \mathcal{O}$. Polyhedra in group $\mathcal{O}$ typically transform into polyhedra in the same group, with $T_{\mathcal{O} \rightarrow \mathcal{O}}=1.6$. Similarly, polyhedra in group $\mathcal{M}$ choose to transform into polyhedra in the same group $\left(T_{\mathcal{M} \rightarrow \mathcal{M}}=1.7\right)$; however, here inter-group transitions are also apparent, with $T_{\mathcal{M} \rightarrow \mathcal{L}}=1.4$ and $T_{\mathcal{L} \rightarrow \mathcal{M}}=1.7$.

The above dynamical analysis further corroborates our earlier observations based on $\hat{W}_{6}^{\prime}$ (static analysis) that polyhedra in groups $\mathcal{J}$ and $\mathcal{K}$ are characterized by a large degree of similarity to polyedra in $\mathcal{I}$. Further, it indicates that polyhedra in groups $\mathcal{K}$ and $\mathcal{L}$ constitute intermediate structures between groups with clear fivefold orientational order (groups $\mathcal{I}$ and $\mathcal{J}$ ) and groups that have no clear fivefold orientational order $(\mathcal{M}$ and $\mathcal{O}$ ). 


\begin{tabular}{ccccccc}
\hline $\mathcal{X}$ & $\mathcal{Y}$ & $\mathcal{J}$ & $\mathcal{K}$ & $\mathcal{L}$ & $\mathcal{M}$ & $\mathcal{O}$ \\
\hline $\mathcal{I}$ & 0 & 0.29 & 0.36 & $<10^{-3}$ & $<10^{-3}$ & $<10^{-2}$ \\
$\mathcal{J}$ & 0.29 & 0.20 & 0.87 & 0.54 & $<10^{-2}$ & 0.65 \\
$\mathcal{K}$ & 0.36 & 0.89 & $\mathbf{2 . 4 3}$ & $\mathbf{2 . 7 1}$ & 0.93 & $\mathbf{4 . 4 0}$ \\
$\mathcal{L}$ & $<10^{-2}$ & 0.54 & $\mathbf{2 . 6 9}$ & $\mathbf{2 . 8 7}$ & $\mathbf{2 . 0 8}$ & $\mathbf{5 . 4 6}$ \\
$\mathcal{M}$ & $<10^{-3}$ & $<10^{-2}$ & 0.95 & $\mathbf{2 . 0 7}$ & $\mathbf{1 . 1 6}$ & $\mathbf{3 . 4 8}$ \\
$\mathcal{O}$ & $<10^{-2}$ & 0.63 & $\mathbf{4 . 4 0}$ & $\mathbf{5 . 4 6}$ & $\mathbf{3 . 5 0}$ & $\mathbf{4 9 . 7 7}$ \\
\hline
\end{tabular}

Table 5: Values of $F_{\mathcal{X} \rightarrow \mathcal{Y}}$ for $T=1300 \mathrm{~K}$. All values are given in \%.

One deficiency of the metric $T_{\mathcal{X} \rightarrow \mathcal{Y}}$ is that it does not describe how important in the total picture of structural transitions any particular transition $\mathcal{X} \rightarrow \mathcal{Y}$ is. This can be remediated by examining the values of $F_{\mathcal{X} \rightarrow \mathcal{Y}}$, which we report in Table 5. Again, we greyed out transitions that occur infrequently, i.e., $\mathcal{I} \rightleftharpoons \mathcal{L}$, $\mathcal{I} \rightleftharpoons \mathcal{M}, \mathcal{I} \rightleftharpoons \mathcal{O}$ and $\mathcal{J} \rightleftharpoons \mathcal{M}$, and $\mathcal{I} \rightarrow \mathcal{I}$. Transitions that occur frequently $\left(F_{\mathcal{X} \rightarrow \mathcal{Y}}>1 \%\right)$ were highlighted in bold. It is those transitions that dictate the total picture of transitions. They encompass all possible

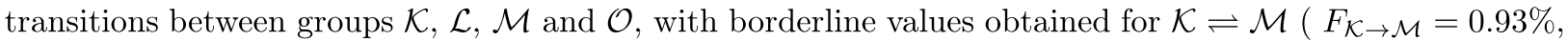
$\left.F_{\mathcal{M} \rightarrow \mathcal{K}}=0.95 \%\right)$. These 14 types of transitions account for almost $95 \%$ of all observed transitions, with transitions involving $\mathcal{O}$ being the most common $(\mathcal{O} \rightarrow \mathcal{O}$ accounts for $\approx 50 \%$, and transitions to and from $\mathcal{O}$ account for almost $27 \%$ of all observed transitions). These values highlight the importance of the group of other polyhedra $(\mathcal{O})$, whose large diversity (over 1000 distinct polyhedra types) indicates that a more detailed analysis of structural transitions in liquid $\mathrm{Cu}$ is next to impossible. Despite the significant simplification offered by classifying polyhedra into groups, detailed characterization can only be performed for about $22 \%$ of all transitions, which are those that take place between well-defined groups $\mathcal{I}$ - $\mathcal{M}$.

A pairwise comparison of $F_{\mathcal{X} \rightarrow \mathcal{Y}}$ yields additional insight into icosahedral ordering in liquid $\mathrm{Cu}$, revealing that icosahedra (polyhedra in $\mathcal{I}$ ) arise mainly through transitions from groups $\mathcal{J}$ and $\mathcal{K}$, and do not form through direct transitions from groups $\mathcal{L}, \mathcal{M}$ or $\mathcal{O}$. In the process of icosahedron forming, the role of groups $\mathcal{J}$ and $\mathcal{K}$ is similarly pronounced, as $F_{\mathcal{J} \rightarrow \mathcal{I}} \approx F_{\mathcal{K} \rightarrow \mathcal{I}}$.

\subsection{Structural correlations}

We will now examine structural correlations and set out to determine which types of polyhedra tend to occur together (connect). We will say that a polyhedron $\mathcal{V}^{\prime}(j)$ is connected to $\mathcal{V}^{\prime}(i)$, if and only if particle $j$ is among the neighbors of particle $i$, i.e., it introduces a face into polyhedron $\mathcal{V}^{\prime}(i)$. If the polyhedra $\mathcal{V}^{\prime}(i)$ and $\mathcal{V}^{\prime}(j)$ belong, respectively, to groups $\mathcal{X}$ and $\mathcal{Y}$, we will denote such connection with $\mathcal{X} \Leftarrow \mathcal{Y}$, and the symbol $C_{\mathcal{X} \Leftarrow \mathcal{Y}}$ will denote the number of observed connections $\mathcal{X} \Leftarrow \mathcal{Y}$. Analogously, the symbol $C_{\mathcal{X}} \Leftarrow \mathcal{A}$ will denote the total number of connections $\mathcal{X} \Leftarrow \mathcal{A}$ made by polyhedra from group $\mathcal{X}$.

In order to quantify structural correlations, we introduce a new measure, which we will term structural affinity. It is defined as follows:

$$
A_{\mathcal{X} \Leftarrow \mathcal{Y}}=\frac{C_{\mathcal{X} \Leftarrow \mathcal{Y}}}{C_{\mathcal{X} \Leftarrow \mathcal{A}}} \times \frac{1}{n(\mathcal{Y})}
$$

The ratio $C_{\mathcal{X} \Leftarrow \mathcal{Y}} / C_{\mathcal{X} \Leftarrow \mathcal{A}}$ informs on the fraction of connections from group $\mathcal{X}$ realized by connections with polyhedra from group $\mathcal{Y}$. The factor $n(\mathcal{Y})^{-1}$ relates the measured frequency of connections $\mathcal{X} \Leftarrow \mathcal{Y}$ to the expected frequency of occurrence of these connections, given by $n(\mathcal{Y})$. This is the frequency that would be observed if all connections were equally probable and if polyhedra from group $\mathcal{X}$ were equally likely to connect to polyhedra from all groups.

The measure $A_{\mathcal{X} \Leftarrow \mathcal{Y}}$ is similar to the previously introduced $T_{\mathcal{X} \rightarrow \mathcal{Y}}$, except it deals with structural correlations. By analysing the values of $A_{\mathcal{X} \Leftarrow \mathcal{Y}}$ it becomes possible to ascertain which polyhedra have an affinity for occurring together. A value of $A_{\mathcal{X} \Leftarrow \mathcal{Y}}>1$ is evidence for an above-average affinity of polyhedra from $\mathcal{X}$ to pair with polyhedra from $\mathcal{Y}$. Conversely, a value of $A_{\mathcal{X}} \Leftarrow \mathcal{Y}<1$ indicates structural incompatibility of polyhedra from groups $\mathcal{X}$ and $\mathcal{Y}$. 


\begin{tabular}{|c|c|c|c|c|c|c|}
\hline$\widehat{\mathcal{X}}$ & $\mathcal{I}$ & $\mathcal{J}$ & $\mathcal{K}$ & $\mathcal{L}$ & $\mathcal{M}$ & $\mathcal{O}$ \\
\hline $\mathcal{I}$ & 2.04 & 1.80 & 1.24 & 0.86 & 0.65 & 0.93 \\
\hline $\mathcal{J}$ & 1.66 & 1.44 & 1.15 & 0.94 & 0.79 & 0.94 \\
\hline $\mathcal{K}$ & 1.19 & 1.19 & 1.06 & 0.98 & 0.89 & 0.98 \\
\hline $\mathcal{L}$ & 0.81 & 0.97 & 0.97 & 1.01 & 1.00 & 1.02 \\
\hline $\mathcal{M}$ & 0.62 & 0.82 & 0.90 & 1.01 & 1.07 & 1.05 \\
\hline $\mathcal{O}$ & 0.81 & 0.90 & 0.90 & 0.94 & 0.96 & 1.08 \\
\hline
\end{tabular}

Table 6: Values of parameters $A_{\mathcal{X} \Leftarrow \mathcal{Y}}$ for $T=1300 \mathrm{~K}$.

An analysis for $T=1300 \mathrm{~K}$ is given in Table 6 . Large values $\left(A_{\mathcal{X} \Leftarrow \mathcal{Y}}>4 / 3\right)$ are shown in bold, and low values $\left(A_{\mathcal{X} \Leftarrow \mathcal{Y}}<3 / 4\right)$ are greyed out.

In general, we observe that icosahedra have a large affinity for other icosahedra. An average icosahedron connects to another icosahedron more often (by a factor of about 2) than it would in the absence of structural correlations. Icosahedra also display an above-average affinity for icosahedron-like structures, as evidenced by $A_{\mathcal{I} \Leftarrow \mathcal{J}}=1.8$ and $A_{\mathcal{J} \Leftarrow \mathcal{I}}=1.66$. A similar trend is observed for polyhedra from $\mathcal{K}$, which also display an above-average affinity for polyhedra from $\mathcal{I}$ and $\mathcal{J}$, although the deviation from average is not as pronounced. Another apparent tendency is for polyhedra from $\mathcal{M}$ to avoid contact with polyhedra from $\mathcal{I}$. This is reflected in the low values of $A_{\mathcal{M} \Leftarrow \mathcal{I}}=0.65$ and $A_{\mathcal{I} \Leftarrow \mathcal{M}}=0.62$, which are the lowest observed. Again, we ascertain the intermediate character of polyhedra from $\mathcal{L}$, which have similar affinities for all other polyhedra groups $\mathcal{J}, \mathcal{K}, \mathcal{L}, \mathcal{M}$ and $\mathcal{O}$ (as seen from values of $A_{\mathcal{L} \Leftarrow \mathcal{A}}$ and $A_{\mathcal{A} \Leftarrow \mathcal{L}}$ close to unity), except for true icosahedra $\mathcal{I}$, which they avoid.

The above analysis suggests that in the structural picture of liquid copper we should be observing a clustering of polyhedra $\mathcal{I}, \mathcal{J}$ and $\mathcal{K}$. This is well-illustrated by the structural visualization shown in Fig. 10, from which it is apparent that these polyhedra indeed tend to cluster together in many-atom formations, with individual polyhedra from these group rarely seen individually.

In order to describe the observed clustering quantitatively, we performed clustering analysis. We will say that a given polyhedron $\mathcal{V}^{\prime}(j)$ is connected to a given cluster when (i) it is connected to any polyhedron $\mathcal{V}^{\prime}(i)$ belonging to that cluster or when (ii) any polyhedron $\mathcal{V}^{\prime}(i)$ belonging to that cluster is connected to polyhedron $\mathcal{V}^{\prime}(j)$

Fig. 11, panel a) shows the frequencies of occurrence of clusters built from polyhedra $\mathcal{I}, \mathcal{J}$ and $\mathcal{K}$, for $T=1300, T=1600$ and $T=1900 \mathrm{~K}$, as fractions of the total number of atoms $N$. For example a value of $0.5 \%$ for a cluster size of 120 informs that in a time-averaged picture $0.5 \%$ of all atoms belong to 120 -atom clusters formed by atoms described with polyhedra from groups $\mathcal{I}, \mathcal{J}$ and $\mathcal{K}$.

At higher temperatures $(1900 \mathrm{~K})$, polyhedra $\mathcal{I}, \mathcal{J}$ and $\mathcal{K}$ most often form small clusters with sizes not larger than 25 atoms. At lower temperatures the tendency to form larger clusters is significantly stronger, and at $1300 \mathrm{~K}$ a pronounced second maximum can be observed, corresponding to large clusters, with sizes between 50 and 150 atoms. At this temperature only $13 \%$ of all $\mathcal{I}, \mathcal{J}$ and $\mathcal{K}$ polyhedra are clustered into small clusters, not larger than 25 atoms.

We will now complement the above analysis by examining how polyhedra in groups $\mathcal{L}, \mathcal{M}$ and $\mathcal{O}$ cluster. Figure 11, panel b) demonstrates that in all temperatures these polyhedra comprise a single large cluster spanning the entire system. 
Figure 10: Clustering of polyhedra from groups $\mathcal{I}$ (blue), $\mathcal{J}$ (orange) and $\mathcal{K}$ (yellow). Pairs of particles whose polyhedra belong to these groups that are not further than $3.5 \AA$ are drawn using bonds. Smaller spheres denote polyhedra from $\mathcal{L}$ (purple), $\mathcal{M}$ (green) and $\mathcal{O}$ (light blue). The visualization has been prepared using OVITO [80].
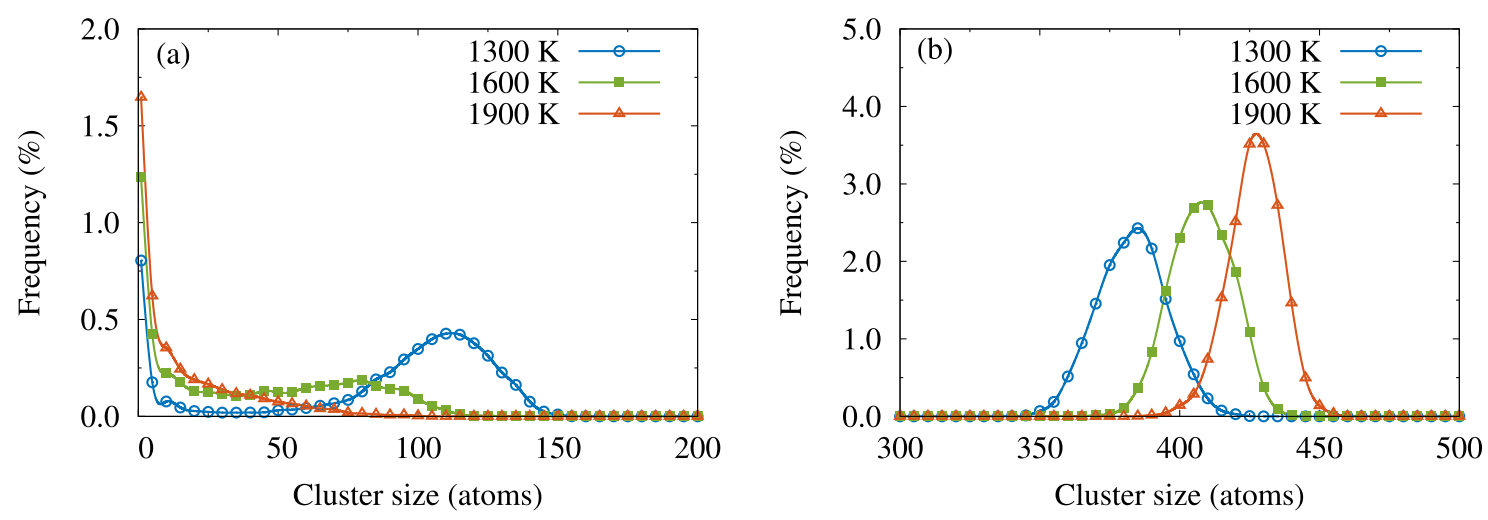

Figure 11: Clustering of polyhedra from groups $\mathcal{I}, \mathcal{J}$ and $\mathcal{K}$ (panel a), and of polyhedra from groups $\mathcal{L}, \mathcal{M}$ and $\mathcal{O}$ (panel b).

The observations made so far allow us to conceptually describe the structure of liquid copper. This structure can be understood as a system of clusters of particles characterized by marked fivefold orientational order (polyhedra in $\mathcal{I}, \mathcal{J}$ and $\mathcal{K}$ ), embedded in a coherent (continuous) matrix of particles that do not display this kind of order (polyhedra in $\mathcal{L}, \mathcal{M}$ and $\mathcal{O}$ ). This icosahedral order manifests increasingly with decreasing temperature - this is reflected by (i) an increase in the number of icosahedron-like polyhedra, and (ii) a significant increase in the size of clusters composed of particles with icosahedral ordering. Close to the melting point the presence of icosahedral ordering is so pronounced that it becomes continuous network of icosahedral structures embedded in a non-icosahedral matrix, with the network practically spanning the entire volume. This is well illustrated by Fig. 10. At $1300 \mathrm{~K}$ we find over $90 \%$ of all polyhedra to either belong to groups $\mathcal{I}, \mathcal{J}$ and $\mathcal{K}$ or to be directly connected to at least one polyhedron from these groups. This demonstrates the importance of icosahedral ordering to elucidating the structure and dynamics of liquid copper. 
Similar conclusions were reached by Celino et al. [20], who ascertained that in undercooled liquid copper the spatial extension of icosahedral ordering involves more than $70 \%$ of the whole undercooled system, describing the structure as large and complex regions with icosahedral ordering embedded in a disordered atomic structure. Tendencies for icosahedra to cluster and form a string-like network structure were observed also in metallic glasses. Shimono et al. showed that this phenomenon exists in liquid and glassy phases of $\mathrm{Zr}_{40} \mathrm{Cu}_{60}$ metallic glasses [52], whereas Xie et al. described it for $\mathrm{TiAl}_{3}$ metallic glasses [58]. Clustering of icosahedra has also been observed experimentally by Leocmach and Tanaka [54], who, by employing confocal microscopy, demonstrated that in colloidal (hard sphere) supercooled liquid [81] icosahedral clusters can be as large as hundreds to thousands of particles.

\section{Discussion}

\subsection{Common neighbor analysis}

The structural analysis undertaken so far in this work relied on a combination of two descriptors: an orientational one (bond-order parameters) and a geometrical one (Voronoi signatures). We showed that their simultaneous application can elucidate a number of structural features related to the presence of icosahedral structure. Below we would like to complement this analysis by employing the CNA approach, in line with the approach of Celino et al., directly comparing with results presented in Ref. [20].

Within the framework of CNA the immediate neighborhood of a particle $i$ is described by characterizing the bonds between it and all its neighbors $j$. For each bond three values are computed, which indicate the number of nearest neighbors common to the central particle $i$ and its neighbor $j\left(n_{\mathrm{cn}}\right)$, the number of bonds between common neighbors $\left(n_{\mathrm{b}}\right)$, and the number of bonds in the longest chain of bonds connecting the common neighbors $\left(n_{\mathrm{lcb}}\right)$. In this formalism, the fcc structure is characterized by the presence of twelve $(4,2,1)$ bonds, bcc is characterized by eight $(6,6,6)$ bonds and six $(4,4,4)$ bonds, hcp has six $(4,2,1)$ bonds and six $(4,2,2)$ bonds, while icosahedral ordering manifests by $(5,5,5)$ bonds, of which there are 12 in a perfect icosahedron.

The above observation is the basis of the methodology of Celino et al., who employed the $\left(n_{\mathrm{cn}}, n_{\mathrm{b}}, n_{\mathrm{lcb}}\right)$ signature to measure the degree of icosahedrity, defining icosahedron-like structures as those having a number of $(5,5,5)$ bonds of at least 6 .

Using the same approach, we analysed the structure of liquid copper at $T=1300 \mathrm{~K}$. When determining the parameters $\left(n_{\mathrm{cn}}, n_{\mathrm{b}}, n_{\mathrm{lcb}}\right)$ we used the geometrical definition of neighbors based on the cleaned up Voronoi diagram. This freed us from having to select a suitable cutoff radius and simultaneously made the structural picture clearer, reducing the number of observed $\left(n_{\mathrm{cn}}, n_{\mathrm{b}}, n_{\mathrm{lcb}}\right)$ signatures.

\begin{tabular}{cccc}
\hline $\begin{array}{c}\text { CNA signature } \\
\left(n_{\mathrm{cn}}, n_{\mathrm{b}}, n_{\mathrm{lcb}}\right)\end{array}$ & $\begin{array}{c}\text { Frequency } \\
(\%)\end{array}$ & $\begin{array}{c}\text { CNA signature } \\
\left(n_{\mathrm{cn}}, n_{\mathrm{b}}, n_{\mathrm{lcb}}\right)\end{array}$ & $\begin{array}{c}\text { Frequency } \\
(\%)\end{array}$ \\
\hline$(3,1,1)$ & 1.9 & $(5,3,2)$ & 2.4 \\
$(3,2,2)$ & 2.3 & $(5,4,3)$ & 20.3 \\
$(4,2,1)$ & 2.5 & $(5,5,5)$ & 25.1 \\
$(4,2,2)$ & 4.8 & $(6,5,4)$ & 2.1 \\
$(4,3,2)$ & 18.7 & $(6,6,6)$ & 9.5 \\
$(4,4,4)$ & 7.7 & & \\
\hline
\end{tabular}

Table 7: Frequencies of occurrence of most common $(>1 \%)$ types of bonds. The enumerated 11 types account for $97.3 \%$ of all bonds observed in liquid copper at $T=1300 \mathrm{~K}$. 
Table 7 enumerates the most common bond types and their frequencies. The overall picture obtained from CNA confirms marked icosahedral ordering. $(5,5,5)$ bonds (typical for icosahedra) and $(5,4,3)$ bonds (typical for distorted icosahedra, originating from the breaking of two bonds in a 5-element chain of common neighbors and the forming of a single new bond) constitute almost half of all bonds. Another marked feature is the presence of topologies typical for the bcc structure, i.e., $(4,4,4)$ bonds and $(6,6,6)$ bonds, accounting for over $17 \%$ of all bonds. A similarly large fraction (almost $19 \%$ ) is accounted by $(4,3,2)$ bonds, which can be understood to be an intermediate form between two motifs: $(4,4,4)$ - characteristic for bcc, and $(4,2,2)$ - characteristic for hcp.

The above analysis clearly demonstrates that topologies typical for icosahedral ordering are ubiquitous in liquid $\mathrm{Cu}$. However, it does not make it clear to what degree $(5,5,5)$ bonds congregate around single central atoms, creating structures with a degree of icosahedrity. In order to clarify this point, we followed Celino et al., determining for each central atom $i$ the value of the parameter $n_{555}(i)$, which expresses the number of $(5,5,5)$ bonds that this atom shares with its neighbors.

In Table 8 we present the frequencies of occurrence of atoms with different values of $n_{555}$. The obtained structural picture is dominated by atoms with $n_{555}<6$, which account for $82 \%$ of all atoms. The most common scenario $(18 \%)$ is for atoms to form two $(5,5,5)$ bonds, followed by $n_{555}=1,3$ and 4 , with frequencies of occurence between $14 \%$ and $16 \%$. Icosahedron-like structures with $n_{555} \geq 6$ account for $18 \%$ of all atoms, with the majority of this contribution being due to atoms forming between 6 ad 8 (5,5,5) bonds (almost 15.5\%). Atoms forming $12(5,5,5)$ bonds are seen to be very scarce $(0.6 \%)$.

A direct comparison of the structural pictures obtained from CNA and through Voronoi analysis runs into difficulties stemming from the differences in the methodology - CNA is a topological approach, while Voronoi analysis is a geometrical one. The fraction of icosahedral structures obtained via CNA by counting atoms with $n_{555} \geq 6$ is $18 \%$ (for $T=1300 \mathrm{~K}$ ). In contrast, the corresponding value obtained from Voronoi analysis as a sum of fractions of $\mathcal{I}$ and $\mathcal{J}$ polyhedra is $5.9 \%$. The additional inclusion of $\mathcal{K}$ polyhedra yields $23.5 \%$, which is still not in good agreement with CNA. This results suggests that the definition of icosahedrity based on $n_{555}$ is markedly different from the one based on Voronoi signatures.

\begin{tabular}{cccccccc}
\hline & Frequency & \multicolumn{7}{c}{ Decomposition (\%) } \\
$n_{555}$ & $(\%)$ & $\mathcal{I}$ & $\mathcal{J}$ & $\mathcal{K}$ & $\mathcal{L}$ & $\mathcal{M}$ & $\mathcal{O}$ \\
\hline 0 & 9.88 & 0.1 & 0.5 & 7.7 & 16.0 & 15.1 & 60.6 \\
1 & 16.06 & 0.2 & 0.9 & 10.0 & 20.0 & 14.4 & 54.5 \\
2 & 17.65 & 0.3 & 1.6 & 13.1 & 21.7 & 13.1 & 50.2 \\
3 & 15.59 & 0.5 & 2.4 & 15.8 & 23.0 & 9.3 & 48.9 \\
4 & 13.85 & 1.2 & 3.9 & 20.7 & 23.1 & 7.8 & 43.3 \\
5 & 8.94 & 1.5 & 4.8 & 25.3 & 17.4 & 1.5 & 49.4 \\
\hline 6 & 7.61 & 1.4 & 7.9 & 28.5 & 20.8 & 1.7 & 39.6 \\
7 & 3.71 & 2.1 & 13.7 & 26.8 & 4.3 & 1.6 & 51.4 \\
8 & 4.15 & 12.3 & 12.7 & 42.4 & 3.3 & 2.0 & 27.2 \\
9 & 0.76 & 0 & 0.3 & 17.6 & 2.3 & 0.2 & 79.5 \\
10 & 1.17 & 0 & 45.0 & 15.5 & 8.5 & 0.1 & 30.8 \\
11 & 0.02 & 0 & 0 & 0 & 0 & 0 & 100 \\
12 & 0.60 & 58.3 & 0 & 14.4 & 0.1 & 0.2 & 26.9 \\
\hline$<6$ & 81.98 & 0.7 & 2.7 & 16.1 & 20.7 & 9.9 & 49.8 \\
$\geq 6$ & 18.02 & 9.0 & 15.1 & 30.3 & 4.0 & 1.4 & 40.1 \\
\hline
\end{tabular}

Table 8: Distribution of atoms with respect to the parameter $n_{555}$ and their classification into groups $\mathcal{I}$ - $\mathcal{M}$ and $\mathcal{O}$. 
In order to investigate this issue in depth we examined the correlations between the parameter $n_{555}(i)$ and the Voronoi signature $\left(f_{3}^{\prime}(i), f_{4}^{\prime}(i), f_{5}^{\prime}(i), f_{6}^{\prime}(i)\right)$. The results of this analysis are included in Table 8 , where for each value of $n_{555}=0,1,2, \ldots, 12$ we decomposed the frequency of occurrence into contributions from polyhedra belonging to groups $\mathcal{I}-\mathcal{M}$ and $\mathcal{O}$. The obtained values only strengthen the impression of distinctness of the two methods - atoms with the same value of $n_{555}$ show broad spectra of Voronoi signatures. This is especially seen for $n_{555}=8$, where there are significant contributions from $\mathcal{I}$ and $\mathcal{J}$ (almost $13 \%$ each), a large contribution from $\mathcal{K}$ (almost 43\%), and a large contribution from other polyhedra $(\mathcal{O})$ (about $27 \%$ ). For all values $n_{555} \geq 6$ (i.e., for atoms deemed icosahedron-like in Celino's approach) we find a marked presence of contributions from $\mathcal{O}-$ e.g., up to $50 \%$ for $n_{555}=7$ and up to $80 \%$ for $n_{555}=9$. Among these same atoms Voronoi analysis distinguishes only $24 \%$ atoms to be icosahedron-like (belonging to $\mathcal{I}$ or $\mathcal{J}$ ), while almost $40 \%$ atoms with $n_{555} \geq 6$ are identified as other (belonging to $\mathcal{O}$ ). This clearly shows that CNA with the $n_{555} \geq 6$ criterion yields a different structural interpretation from Voronoi analysis.

To elucidate which of the two approaches yields structures that are characterized by higher degrees of icosahedrity, we grouped the atoms into the following four groups:

1. polyhedra from groups $\mathcal{I}$ - $\mathcal{J}$, simultaneously with $n_{555} \geq 6$,

2. polyhedra from group $\mathcal{O}$, simultaneously with $n_{555}<6$,

3. polyhedra from groups $\mathcal{I}$ - $\mathcal{J}$, simultaneously with $n_{555}<6$,

4. polyhedra from group $\mathcal{O}$, simultaneously with $n_{555} \geq 6$.

The first two groups encompass atoms where the two methods agree to whether the structure is (group 1) or is not (group 2) icosahedral. Conversely, groups 3 and 4 encompass atoms where the two approaches disagree on the icosahedral character.

For $T=1300 \mathrm{~K}$ the populations of the four groups were $n_{1}=4.3 \%, n_{2}=41 \%, n_{3}=2.8 \%$ and $n_{4}=7.2 \%$.

In Fig. 12 we present the distribution of valence angles (panel a) and of the $\hat{W}_{6}^{\prime}$ parameter (panel b) decomposed into the four groups. For the angle distribution of group 1 we observe two pronounced peaks corresponding to the typical icosahedral valence angles of 60 and 108 degrees. The values of $\hat{W}_{6}^{\prime}$ for this group are almost universally negative (with a mean of -0.0955). Group 2 displays a marked fourfold symmetry, with typical angles of 45 and 90 degrees, and the values of $\hat{W}_{6}^{\prime}$ are much closer to zero (with a mean of $-0.0360)$. This demonstrates that groups 1 and 2 indeed encompass structures with, respectively, the largest and lowest degree of icosahedrity.
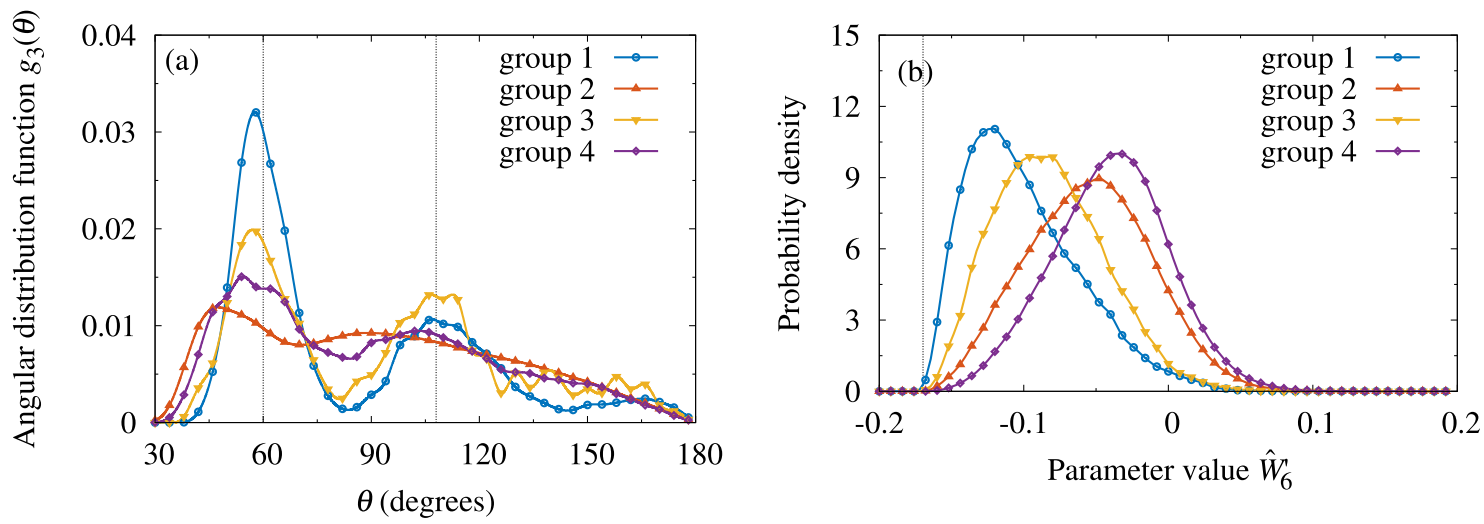

Figure 12: Distributions of valence angles (panel a) and the $\hat{W}_{6}^{\prime}$ parameter (panel b) for groups 1-4 (see text). Dashed vertical lines denote angles typical for icosahedral ordering (60 and 108 degrees) and the value of $\hat{W}_{6}^{\prime}=-0.169754$ corresponding to a perfect icosahedron. 
We will now attempt to elucidate the source of the differences between the CNA approach and Voronoi analysis by examining the characteristics of topologies where the two approaches disagree, i.e., atoms in groups 3 and 4 . Fig. 12 reveals that structures in group 3 (deemed icosahedral by Voronoi analysis only) contribute much more often to angles close to 108 degrees and much less to angles close to 90 degrees, compared to structures in group 4 (deemed icosahedral by CNA only). Similarly the peak at 60 degrees is more pronounced for group 3. This demonstrates that atoms in group 3 display a larger degree of icosahedral ordering compared to atoms in group 4. The same can be inferred from examining the distribution of $\hat{W}_{6}^{\prime}$. Atoms in group 3 are characterized by lower (more negative) values, with a mean of -0.0779 (compared to -0.0507 for group 4).

The above analysis leads us to believe that CNA with a fixed criterion based on $n_{555}$ is not as efficient as Voronoi analysis for identifying icosahedral ordering and that the use of a relatively simple criterion $n_{555} \geq 6$ can lead not only to excluding important information, but even to wrong conclusions. For instance, $n_{3} /\left(n_{1}+n_{3}\right)=40 \%$ and $n_{4} /\left(n_{1}+n_{4}\right)=62 \%$, which means that, for the system under study here, by using CNA one loses information on $40 \%$ of icosahedron-like structures, while among structures classified as icosahedral the majority (over $60 \%$ ) are false positives.

The underlying cause for this limitation is, in our belief, the way the parameter $n_{555}$ is constructed. Since it is discrete in nature, it does not (in the quantitative sense) sufficiently well describe the influence of structural distortions as long as the distortion does not change the topology of bonds. Moreover, the parameter $n_{555}(i)$ does not carry any information on valence angles that the central atom forms with its nearest neighbors. The Voronoi polyhedra approach turns out to be superior in this aspect. While the Voronoi signature is also discrete, it is constructed by geometrical means, and as such it explicitly takes into account distances to nearest neighbors and valence angles. This makes the analysis of the coordination polyhedron a more sensitive method.

\subsection{Transformations of polyhedra}

We will now compare our results to those of Pan et al. [24], who studied the crystallization pathways in iron and also used Voronoi analysis. They showed that the transformations of $\left(0, f_{4}, f_{5}, f_{6}, 0, \ldots\right)$ polyhedra observed during crystallization can be characterized using two transformation styles: $\mathrm{T}_{1}$ and $\mathrm{T}_{2}$. These styles are schematically shown in Fig. 13. In $\mathrm{T}_{1}$ a neigboring atom approaching a central atom introduces a new quadrilateral face at the point of intersection of four faces, increasing the coordination number of the central atom by one. As a result the degeneracy (number of edges) of two faces is also increased by one. In contrast, during the transformation $\mathrm{T}_{2}$ the coordination number remains unchanged; however, the collective motion of nearest neighbors leads to a change in the degeneracy of four faces, which is increased or decreased by one for two pairs of faces.

(a)

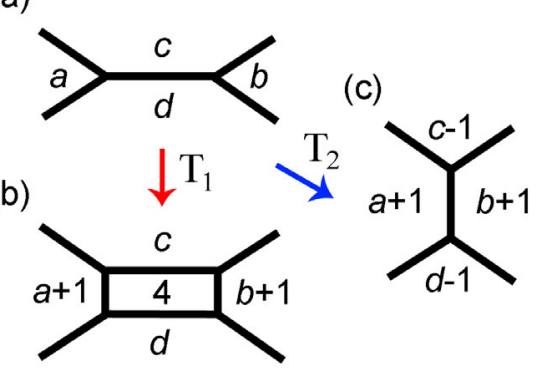

Figure 13: Two transformation styles $-\mathrm{T}_{1}(\mathrm{a}-\mathrm{b})$ and $\mathrm{T}_{2}(\mathrm{a}-\mathrm{c})$. Reproduced following Ref. [24]. 
Pan et al. [24] dinstinguished the following nine transformation patterns for the styles $\mathrm{T}_{1}$ and $\mathrm{T}_{2}$ :

$$
\begin{array}{ll}
\mathrm{T}_{1,1}: & \stackrel{(4,4, c, d)}{\longrightarrow}\left(0, f_{4}-1, f_{5}+2, f_{6}\right), \\
\mathrm{T}_{1,2}: & \stackrel{(4,5, c, d)}{\longrightarrow}\left(0, f_{4}, f_{5}, f_{6}+1\right), \\
\mathrm{T}_{1,3}: & \stackrel{(5,5, c, d)}{\longrightarrow}\left(0, f_{4}+1, f_{5}-2, f_{6}+2\right), \\
\mathrm{T}_{2,1}: & \stackrel{(5,5,5,5)}{\longrightarrow}\left(0, f_{4}+2, f_{5}-4, f_{6}+2\right), \\
\mathrm{T}_{2,2}: & \stackrel{(4,4,6,6)}{\longrightarrow}\left(0, f_{4}-2, f_{5}+4, f_{6}-2\right), \\
\mathrm{T}_{2,3}: & \stackrel{(5,5,5,6)}{\longrightarrow}\left(0, f_{4}+1, f_{5}-2, f_{6}+1\right), \\
\mathrm{T}_{2,4}: & \stackrel{(4,5,6,6)}{\longrightarrow}\left(0, f_{4}-1, f_{5}+2, f_{6}-1\right), \\
\mathrm{T}_{2,5}: & \stackrel{(4,5,5,5)}{\longrightarrow}\left(0, f_{4}+1, f_{5}-2, f_{6}+1\right), \\
\mathrm{T}_{2,6}: & \stackrel{(4,4,5,6)}{\longrightarrow}\left(0, f_{4}-1, f_{5}+2, f_{6}-1\right) .
\end{array}
$$

Each of the above transformations $\mathrm{T}_{t, u}$ (where $t$ indexes styles, and $u$ distinguishes patterns within a style) is characterized by a vector $(a, b, c, d)$ describing the transformation (four integer numbers $a-d$ corresponding to the degeneracy of the faces taking part in the transformation) and the resultant (post-transformation) Voronoi signature.

The first three transformation patterns are based on style $\mathrm{T}_{1}$, while the subsequent six patterns are based on $\mathrm{T}_{2}$. For a transformation with $t=1$ it is possible to define an inverse transformation, which proceeds from right to left and results in the vanishing of one quadrilateral face and a decrease of the degeneracy of two faces by one. We will use the notation $-\mathrm{T}_{t, u}$ to denote a transformation that is the inverse of $\mathrm{T}_{t, u}$. The patterns based on $\mathrm{T}_{2}$ can be grouped into three pairs of inverse transformations, i.e., $\mathrm{T}_{2,1}=-\mathrm{T}_{2,2}$, $\mathrm{T}_{2,3}=-\mathrm{T}_{2,4}$ and $\mathrm{T}_{2,5}=-\mathrm{T}_{2,6}$. It is worth pointing out that the pairs $\mathrm{T}_{2,3}$ and $\mathrm{T}_{2,5}$, and the pairs $\mathrm{T}_{2,4}$ and $\mathrm{T}_{2,6}$ have an identical effect on the Voronoi signature.

Ref. [24] demonstrates that the above nine transformation patterns are sufficient for characterizing the most significant structural transformations observed during the crystallization/melting of iron. With the aim of comparing the general picture of structural transformations in liquid copper with the conclusions obtained by Pan et al. for iron, we calculated the frequencies of occurrence of the above types of transformations and we determined their corresponding patterns. Our analysis was limited to the twelve polyhedra that were considered in Ref. [24] and as such does not include signatures $(1,0,9,3),(0,2,8,1),(0,3,6,2)$ and $(0,4,4,3)$.

Tables 9 and 10 list all transformations occurring betwen the considered 12 types of polyhedra. All results correspond to $T=1300 \mathrm{~K}$. For each polyhedron type we list the corresponding transformations, together with their frequency of occurrence, relative to the total number of transformations observed for the particular polyhedron. For identified transformation we also included the symbol of its corresponding pattern $\mathrm{T}_{t, u}$. To facilitate easier comparison, we highlighted the transformations that Ref. [24] identifies as commonly occurring.

Our analysis shows that the patterns (18)-(26) exhaustively characterize all transformations of considered polyhedra $\left(0, f_{4}, f_{5}, f_{6}, 0, \ldots\right)$. Moreover, for as many as seven out of twelve polyhedra, viz. for $(0,0,12,0)$, $(0,1,10,2),(0,1,10,3),(0,2,8,2),(0,2,8,3),(0,3,6,3)$ and $(0,4,4,6)$, all the transformations identified by Pan et al. as most commonly occurring for iron similarly turned out to be the most abundant in liquid copper. For the remaining five polyhedra the agreement is still qualitatively good, with transformations identified in Ref. [24] as most commonly occurring featured among those occurring commonly in liquid copper.

The above demonstrates that structural transformations in liquid copper and liquid iron are conceptually similar, with somewhat larger variety of possible transformations featuring in liquid $\mathrm{Cu}$. This is evidenced by a larger number of observed transformations ( 65 vs. 51 for iron), and smaller differences in the frequencies of occurrence measured for individual polyhedra. 


\begin{tabular}{|c|c|c|c|}
\hline \multicolumn{2}{|c|}{$\begin{array}{l}\text { Voronoi signature } \\
\qquad\left(f_{3}, f_{4}, f_{5}, f_{6}\right)\end{array}$} & \multirow{2}{*}{$\begin{array}{c}\text { Transformation } \\
\text { pattern } \\
\mathrm{T}_{t, u}\end{array}$} & \multirow{2}{*}{$\begin{array}{c}\text { Relative } \\
\text { frequency } \\
\text { (\%) }\end{array}$} \\
\hline initial & final & & \\
\hline \multirow[t]{2}{*}{$(0,0,12,0)$} & $(0,2,8,2)$ & $\mathrm{T}_{2,1}$ & 52.1 \\
\hline & $(0,1,10,2)$ & $\mathrm{T}_{1,3}$ & 26.9 \\
\hline \multirow[t]{6}{*}{$(0,1,10,2)$} & $(0,3,6,4)$ & $\mathrm{T}_{2,1}$ & 23.5 \\
\hline & $(0,2,8,4)$ & $\mathrm{T}_{1,3}$ & 14.7 \\
\hline & $(\mathbf{0}, \mathbf{2}, \mathbf{8}, \mathbf{3})$ & $\mathrm{T}_{2,3}, \mathrm{~T}_{2,5}$ & 11.1 \\
\hline & $(\mathbf{0}, \mathbf{0}, \mathbf{1 2}, \mathbf{0})$ & $-\mathrm{T}_{1,3}$ & 8.4 \\
\hline & $(\mathbf{0}, \mathbf{1}, \mathbf{1 0}, \mathbf{3})$ & $\mathrm{T}_{1,2}$ & 7.0 \\
\hline & $(0,2,8,2)$ & $-\mathrm{T}_{1,1}$ & 6.3 \\
\hline \multirow[t]{4}{*}{$(0,1,10,3)$} & $(0,2,8,4)$ & $\mathrm{T}_{2,3}, \mathrm{~T}_{2,5}$ & 21.6 \\
\hline & $(\mathbf{0}, \mathbf{1}, \mathbf{1 0}, \mathbf{2})$ & $-\mathrm{T}_{1,2}$ & 14.5 \\
\hline & $(0,3,6,5)$ & $\mathrm{T}_{2,1}$ & 13.4 \\
\hline & $(0,2,8,3)$ & $-\mathrm{T}_{1,1}$ & 1.2 \\
\hline \multirow[t]{6}{*}{$(0,2,8,2)$} & $(\mathbf{0}, \mathbf{3}, \mathbf{6}, \mathbf{4})$ & $\mathrm{T}_{1,3}$ & 13.0 \\
\hline & $(\mathbf{0}, \mathbf{3}, \mathbf{6}, \mathbf{3})$ & $\mathrm{T}_{2,3}, \mathrm{~T}_{2,5}$ & 12.1 \\
\hline & $(\mathbf{0}, \mathbf{4}, \mathbf{4}, \mathbf{4})$ & $\mathrm{T}_{2,1}$ & 9.9 \\
\hline & $(\mathbf{0}, \mathbf{2}, \mathbf{8}, \mathbf{3})$ & $\mathrm{T}_{1,2}$ & 9.6 \\
\hline & $(0,0,12,0)$ & $\mathrm{T}_{2,2}$ & 8.9 \\
\hline & $(0,1,10,2)$ & $\mathrm{T}_{1,1}$ & 3.8 \\
\hline \multirow[t]{7}{*}{$(0,2,8,3)$} & $(\mathbf{0}, 2,8,2)$ & $-\mathrm{T}_{1,2}$ & 15.5 \\
\hline & $(\mathbf{0}, \mathbf{3}, \mathbf{6}, \mathbf{4})$ & $\mathrm{T}_{2,3}, \mathrm{~T}_{2,5}$ & 15.0 \\
\hline & $(\mathbf{0}, \mathbf{1}, \mathbf{1 0}, \mathbf{2})$ & $\mathrm{T}_{2,4}, \mathrm{~T}_{2,6}$ & 11.0 \\
\hline & $(\mathbf{0}, \mathbf{3}, \mathbf{6}, \mathbf{5})$ & $\mathrm{T}_{1,3}$ & 8.9 \\
\hline & $(0,2,8,4)$ & $\mathrm{T}_{1,2}$ & 8.8 \\
\hline & $(0,4,4,5)$ & $\mathrm{T}_{2,1}$ & 5.8 \\
\hline & $(0,3,6,3)$ & $-\mathrm{T}_{1,1}$ & 3.7 \\
\hline \multirow[t]{6}{*}{$(0,2,8,4)$} & $(0,1,10,2)$ & $-\mathrm{T}_{1,3}$ & 11.2 \\
\hline & $(0,3,6,5)$ & $\mathrm{T}_{2,3}, \mathrm{~T}_{2,5}$ & 9.8 \\
\hline & $(\mathbf{0}, \mathbf{1}, \mathbf{1 0}, \mathbf{3})$ & $\mathrm{T}_{2,4}, \mathrm{~T}_{2,6}$ & 7.2 \\
\hline & $(0,4,4,6)$ & $\mathrm{T}_{2,1}$ & 7.0 \\
\hline & $(\mathbf{0}, \mathbf{3}, \mathbf{6}, \mathbf{4})$ & $-\mathrm{T}_{1,1}$ & 6.9 \\
\hline & $(0,2,8,3)$ & $-\mathrm{T}_{1,2}$ & 6.9 \\
\hline
\end{tabular}

Table 9: Observed transformations of Voronoi polyhedra (for groups $\mathcal{I}, \mathcal{J}$ and $\mathcal{K}$ ), and their frequencies of occurrence $(T=1300 \mathrm{~K})$. Two, comma-separated $\mathrm{T}_{t, u}$ symbols are given whenever a transformation can be realized via two patterns. 


\begin{tabular}{|c|c|c|c|}
\hline \multicolumn{2}{|c|}{$\begin{array}{l}\text { Voronoi signature } \\
\quad\left(f_{3}, f_{4}, f_{5}, f_{6}\right)\end{array}$} & $\begin{array}{c}\text { Transformation } \\
\text { pattern } \\
\mathrm{T}_{t, u}\end{array}$ & $\begin{array}{c}\text { Relative } \\
\text { frequency } \\
(\%)\end{array}$ \\
\hline$(0,3,6,3)$ & $\begin{array}{c}(\mathbf{0}, \mathbf{3}, \mathbf{6}, \mathbf{4}) \\
(\mathbf{0}, \mathbf{2}, \mathbf{8}, \mathbf{2}) \\
(0,4,4,5) \\
(0,4,4,4) \\
(0,2,8,3)\end{array}$ & $\begin{array}{c}\mathrm{T}_{1,2} \\
\mathrm{~T}_{2,4}, \mathrm{~T}_{2,6} \\
\mathrm{~T}_{1,3} \\
\mathrm{~T}_{2,3}, \mathrm{~T}_{2,5} \\
\mathrm{~T}_{1,1}\end{array}$ & $\begin{array}{c}\mathbf{1 8 . 5} \\
\mathbf{1 2 . 2} \\
6.2 \\
6.0 \\
2.3\end{array}$ \\
\hline$(0,3,6,4)$ & $\begin{array}{c}(\mathbf{0}, \mathbf{3}, \mathbf{6}, \mathbf{3}) \\
(\mathbf{0}, \mathbf{3}, \mathbf{6}, \mathbf{5}) \\
(\mathbf{0}, \mathbf{1}, \mathbf{1 0}, \mathbf{2}) \\
(\mathbf{0}, \mathbf{2}, \mathbf{8}, \mathbf{2}) \\
(0,4,4,5) \\
(0,2,8,3) \\
(\mathbf{0}, \mathbf{4}, \mathbf{4}, \mathbf{6}) \\
(0,4,4,4) \\
(0,2,8,4)\end{array}$ & $\begin{array}{c}-\mathrm{T}_{1,2} \\
\mathrm{~T}_{1,2} \\
\mathrm{~T}_{2,2} \\
-\mathrm{T}_{1,3} \\
\mathrm{~T}_{2,3}, \mathrm{~T}_{2,5} \\
\mathrm{~T}_{2,4}, \mathrm{~T}_{2,6} \\
\mathrm{~T}_{1,3} \\
-\mathrm{T}_{1,1} \\
\mathrm{~T}_{1,1}\end{array}$ & $\begin{array}{r}\mathbf{1 1 . 8} \\
\mathbf{9 . 1} \\
\mathbf{8 . 6} \\
\mathbf{8 . 2} \\
6.7 \\
5.8 \\
\mathbf{5 . 2} \\
3.9 \\
3.4\end{array}$ \\
\hline$(0,3,6,5)$ & $\begin{array}{c}(\mathbf{0}, \mathbf{3}, \mathbf{6}, \mathbf{4}) \\
(0,2,8,4) \\
(\mathbf{0}, \mathbf{4}, \mathbf{4}, \mathbf{6}) \\
(0,2,8,3) \\
(0,1,10,3) \\
(0,4,4,5)\end{array}$ & $\begin{array}{c}-\mathrm{T}_{1,2} \\
\mathrm{~T}_{2,4}, \mathrm{~T}_{2,6} \\
\mathrm{~T}_{2,3}, \mathrm{~T}_{2,5} \\
-\mathrm{T}_{1,3} \\
\mathrm{~T}_{2,2} \\
-\mathrm{T}_{1,1}\end{array}$ & $\begin{array}{r}\mathbf{1 9 . 4} \\
10.0 \\
\mathbf{7 . 9} \\
7.0 \\
5.2 \\
2.3\end{array}$ \\
\hline$(0,4,4,4)$ & $\begin{array}{c}(\mathbf{0}, \mathbf{2}, \mathbf{8}, \mathbf{2}) \\
(\mathbf{0}, \mathbf{3}, \mathbf{6}, \mathbf{4}) \\
(0,3,6,3) \\
(\mathbf{0}, \mathbf{4}, \mathbf{4}, \mathbf{5})\end{array}$ & $\begin{array}{c}\mathrm{T}_{2,2} \\
\mathrm{~T}_{1,1} \\
\mathrm{~T}_{2,4}, \mathrm{~T}_{2,6} \\
\mathrm{~T}_{1,2}\end{array}$ & $\begin{array}{c}\mathbf{1 7 . 0} \\
\mathbf{1 0 . 1} \\
9.6 \\
\mathbf{8 . 0}\end{array}$ \\
\hline$(0,4,4,5)$ & $\begin{array}{l}(\mathbf{0}, \mathbf{3}, \mathbf{6}, \mathbf{4}) \\
(0,3,6,3) \\
(\mathbf{0}, \mathbf{4}, \mathbf{4}, \mathbf{6}) \\
(0,4,4,4) \\
(0,2,8,3) \\
(0,3,6,5)\end{array}$ & $\begin{array}{c}\mathrm{T}_{2,4}, \mathrm{~T}_{2,6} \\
-\mathrm{T}_{1,3} \\
\mathrm{~T}_{1,2} \\
-\mathrm{T}_{1,2} \\
\mathrm{~T}_{2,2} \\
\mathrm{~T}_{1,1}\end{array}$ & $\begin{array}{r}\mathbf{1 7 . 9} \\
10.9 \\
\mathbf{9 . 3} \\
8.9 \\
6.1 \\
2.9\end{array}$ \\
\hline$(0,4,4,6)$ & $\begin{array}{l}(0,3,6,4) \\
(0,3,6,5) \\
(0,4,4,5) \\
(0,2,8,4)\end{array}$ & $\begin{array}{c}-\mathrm{T}_{1,3} \\
\mathrm{~T}_{2,4}, \mathrm{~T}_{2,6} \\
-\mathrm{T}_{1,2} \\
\mathrm{~T}_{2,2}\end{array}$ & $\begin{array}{l}16.2 \\
11.6 \\
10.9 \\
10.7\end{array}$ \\
\hline
\end{tabular}

Table 10: Observed transformations of Voronoi polyhedra - cont'd (groups $\mathcal{L}$ and $\mathcal{M}$ ). 


\section{Conclusions}

In this work we employed a tight-binding molecular dynamics approach to simulate liquid $\mathrm{Cu}$ in a range of temperatures between $1300 \mathrm{~K}$ and $1900 \mathrm{~K}$. By examining standard descriptors of averaged structure (pair correlation functions, angular distribution functions and distributions of $\hat{W}_{6}$ ) we demonstrated that our simulation protocol yields credible structures of liquid $\mathrm{Cu}$, which are in very good agreement with other results - both experimental (due to Di Cicco et al. [9]), and computational (first-principles simulations by Ganesh and Widom [22]). By using a novel approach to structural analysis, which combines Voronoi analysis with a recently proposed formulation [76] of the bond-orientational order parameters method, we performed an in-depth characterization of the icosahedral ordering present in this system.

We characterized local structures present in the liquid state, with differing Voronoi signatures $\left(f_{3}^{\prime}, f_{4}^{\prime}, f_{5}^{\prime}, f_{6}^{\prime}\right)$, with regard to five-fold ordering. To that end we defined and employed a new parameter, $\hat{W}_{6}^{\prime}$, which can be seen - in the context of Ref. [76] - as a natural generalization of the well-known cubic invariant due to Steinhardt, $\hat{W}_{6}$, commonly used in studies of icosahedral ordering. By examining structures with highly negative values of $\hat{W}_{6}^{\prime}$, we showed that apart from structures with the Voronoi signature $(0,0,12,0)$ (perfect icosahedra), structures with signatures $(1,0,9,3)$ and $(0,1,10,2)$, corresponding to defected icosahedra, and structures with signatures $(0,1,10,3)$ and $\left(0,2,8, f_{6}\right)$ (where $\left.f_{6}=1, \ldots, 4\right)-$ since they too display highly negative values of $\hat{W}_{6}^{\prime}$ - can also be seen as icosahedron-like.

We determined the temperature dependence of the frequency of occurrence of particular Voronoi polyhedra, showing that this dependence is well-described by the Arrhenius relation. We showed that, as the temperature approaches the solidification temperature, icosahedron-like polyhedra experience the largest increase in the frequency of occurrence. We calculated the mean energies of formation of different types of polyhedra, showing that they correlate with the $\hat{W}_{6}^{\prime}$ parameter, and are largest for icosahedron-like polyhedra.

By analyzing how polyhedra transform, we determined distributions of their lifetimes, showing that the probability of a polyhedron persisting for a given time is well-described by the exponential distribution. We also showed that the mean lifetime of icosahedra to be the longest, approximately twice that of the global average lifetime. The temperature dependence of the mean lifetime was also found to be well-described by the Arrhenius relation. Furthermore, by examining mean descriptors of transformations (that we introduced in this work), we characterized structural changes in liquid $\mathrm{Cu}$, demonstrating, inter alia, that icosahedra and icosahedron-like polyhedra preferentially transform into one another, and that polyhedra with a low degree of icosahedrity almost never transform into icosahedra.

Furthermore, we performed structural correlation analysis, by examining ways in which different polyhedra connect with one another, demonstrating that icosahedra and icosahedron-like polyhedra preferentially connect and show a tendency towards clustering. We showed that this tendency becomes more marked as the temperature is lowered, and close to the solidification temperature it becomes sufficiently pronounced for almost $90 \%$ of atoms to be directly involved in establishing icosahedral ordering, i.e., being themselves icosahedron-like polyhedra or having icosahedron-like polyhedra as nearest neighbors.

We confronted the approach proposed in this work with common-neighbor analysis (CNA), a well-known method for structural analysis, which has been used several times to characterize icosahedral ordering. We showed that CNA can yield picture of icosahedral ordering that is not entirely credible, and elucidated the reasons for the observed deficiencies of this approach. Finally, we related the structural transformations observed in liquid $\mathrm{Cu}$ to corresponding structural transformations in liquid Fe [24], showing that - despite some quantitative differences - significant similarities exist between these systems.

Results presented in this work not only strengthen the notion of strongly pronounced icosahedral ordering in liquid $\mathrm{Cu}$, but significantly elucidate its character, supplementing current understanding with novel aspects regarding the types of icosahedron-like structures present, their lifetimes and relative stability, and observed structural correlations. We trust that the new measures and descriptors introduced in this work, the constructs built upon them, and the path we took in our analysis can be successfully, and practically forthwith, employed in studies of icosahedral ordering in other systems, for example in metallic glasses, which have recently experienced an increase in research interest. Such use of the approach we propose would, however, necessitate introducing certain generalizations to account for the fact that multi-component 
systems often feature atoms spanning a wide range of sizes. We believe that this issue could be circumvented by using weighted Voronoi tessellation (also known as radical tessellation). This has been demonstrated in, for instance, Refs. [82-84].

\section{Appendix}

With the aim of further supporting the choice of the face elimination parameter $\alpha_{\mathrm{sf}}$ (Sec. 4.2), we verified how this value affects the mean lifetimes $\left\langle t_{\text {life }}\right\rangle$ presented in Sec. 4.5. It is clear that if no faces are eliminated $\left(\alpha_{\mathrm{sf}}=0\right)$, the calculated lifetimes (and so their mean values) would be underestimated, since the lifetimes of polyhedra would be prematurely terminated by artifact neighbor particles appearing due to thermal vibrations. Setting a non-zero, but small, value for $\alpha_{\text {sf }}$ will eliminate excessively small "artifact" faces, leading to an increase in the observed mean lifetimes. Of course, excessive elimination will bring about the reverse effect - eliminating "reasonable" faces will also prematurely terminate lifetimes, leading to a decrease in $\left\langle t_{\text {life }}\right\rangle$. A suitable value of $\alpha_{\text {sf }}$ can thus be found by looking for the plateau, where the mean lifetimes are largely insensitive to the value of $\alpha_{\mathrm{sf}}$. In this plateau it will be desirable to choose a value from the right, as this will allow for a better pruning of the Voronoi diagram and to classify the neighborhoods of a larger fraction of atoms.

In Fig. 14 we present example mean lifetimes $\left\langle t_{\text {life }}\right\rangle$ as a function of $\alpha_{\text {sf }}$. It can be seen that the lifetimes' behavior follows our reasoning presented above. As $\alpha_{\mathrm{sf}}$ is increased from 0 to $0.02-0.05$ (depending on polyhedron type) the lifetimes increase, practically plateauing in the interval $0.075-0.01$, and systematically decreasing for $\alpha_{\mathrm{sf}}>0.1$. This analysis suggests that the value chosen in this work $\left(\alpha_{\mathrm{sf}}=0.075\right)$ is suitable, serving to eliminate artifact faces, without excessive pruning of the faces that we want retained.

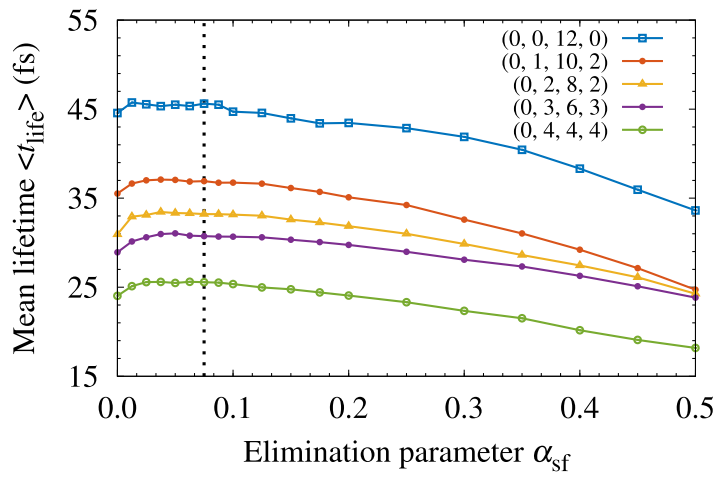

Figure 14: Effect of the parameter $\alpha_{\mathrm{sf}}$ used in the face elimination procedure on the calculated mean lifetimes $\left\langle t_{\text {life }}\right\rangle$, for $T=1300 \mathrm{~K}$, for selected polyhedra. Results for other temperatures and other polyhedra are qualitatively the same.

\section{Acknowledgments}

We acknowledge the support of the Polish Ministry of Science and Higher Education (grant IP2012 043972) and of the TASK Academic Computer Centre (Gdańsk, Poland). This research was also supported in part by the PL-Grid Infrastructure (grant POIG.02.03.00-00-096/10).

\section{Data availability}

The datasets generated during and/or analysed during the current study are available from the corresponding author on reasonable request. 

neering Sciences 215 (1120) (1952) 43-46. doi:10.1098/rspa.1952.0194

[2] D. Turnbull, Formation of crystal nuclei in liquid metals, Journal of Applied Physics 21 (10) (1950) $1022-1028$. doi:10.1063/1.1699435

[3] D. Turnbull, Kinetics of solidification of supercooled liquid mercury droplets, The Journal of Chemical Physics 20 (3) (1952) 411-424. doi:http://dx.doi.org/10.1063/1.1700435.

URL http://scitation.aip.org/content/aip/journal/jcp/20/3/10.1063/1.1700435

[4] P. J. Steinhardt, D. R. Nelson, M. Ronchetti, Icosahedral bond orientational order in supercooled liquids, Phys. Rev. Lett. 47 (1981) 1297-1300. doi:10.1103/PhysRevLett.47.1297. URL http://link.aps.org/doi/10.1103/PhysRevLett.47.1297

[5] P. J. Steinhardt, D. R. Nelson, M. Ronchetti, Bond-orientational order in liquids and glasses, Phys. Rev. B 28 (1983) 784-805. doi:10.1103/PhysRevB.28.784. URL http://link.aps.org/doi/10.1103/PhysRevB.28.784

[6] S. Nosé, F. Yonezawa, Isothermal-isobaric computer simulations of melting and crystallization of a Lennard-Jones system, The Journal of Chemical Physics 84 (3) (1986) 1803-1814. doi:10.1063/1.450427.

[7] H. Jónsson, H. C. Andersen, Icosahedral ordering in the Lennard-Jones liquid and glass, Phys. Rev. Lett. 60 (1988) 22952298. doi:10.1103/PhysRevLett.60.2295. URL https://link.aps.org/doi/10.1103/PhysRevLett.60.2295

[8] T. Schenk, D. Holland-Moritz, V. Simonet, R. Bellissent, D. M. Herlach, Icosahedral short-range order in deeply undercooled metallic melts, Phys. Rev. Lett. 89 (2002) 075507. doi:10.1103/PhysRevLett.89.075507. URL https://link.aps.org/doi/10.1103/PhysRevLett.89.075507

[9] A. Di Cicco, A. Trapananti, S. Faggioni, A. Filipponi, Is there icosahedral ordering in liquid and undercooled metals?, Phys. Rev. Lett. 91 (2003) 135505. doi:10.1103/PhysRevLett.91.135505. URL http://link.aps.org/doi/10.1103/PhysRevLett.91.135505

[10] S. M. Foiles, J. B. Adams, Thermodynamic properties of fcc transition metals as calculated with the embedded-atom method, Phys. Rev. B 40 (1989) 5909-5915. doi:10.1103/PhysRevB.40.5909. URL https://link.aps.org/doi/10.1103/PhysRevB.40.5909

[11] T. Arai, I. Yokoyama, Y. Waseda, A molecular dynamics study of liquid copper near the melting point, Journal of NonCrystalline Solids 117 (1990) 96 - 99. doi:http://dx.doi.org/10.1016/0022-3093(90)90887-R.

URL http://www.sciencedirect.com/science/article/pii/002230939090887R

[12] S. M. Foiles, Application of the embedded-atom method to liquid transition metals, Phys. Rev. B 32 (1985) $3409-3415$. doi:10.1103/PhysRevB.32.3409. URL https://link.aps.org/doi/10.1103/PhysRevB.32.3409

[13] J. M. Holender, Molecular dynamics studies of solid and liquid copper using the Finnis-Sinclair many-body potential, Journal of Physics: Condensed Matter 2 (5) (1990) 1291. URL http://stacks.iop. org/0953-8984/2/i=5/a=020

[14] J. Mei, J. W. Davenport, G. W. Fernando, Analytic embedded-atom potentials for fcc metals: Application to liquid and solid copper, Phys. Rev. B 43 (1991) 4653-4658. doi:10.1103/PhysRevB.43.4653. URL https://link.aps.org/doi/10.1103/PhysRevB.43.4653

[15] T. M. Brown, J. B. Adams, EAM calculations of the thermodynamics of amorphous copper, Journal of Non-Crystalline Solids 180 (2) (1995) 275 - 284. doi:https://doi.org/10.1016/0022-3093(94)00469-2. URL http://www.sciencedirect.com/science/article/pii/0022309394004692

[16] B. Sadigh, G. Grimvall, Molecular-dynamics study of thermodynamical properties of liquid copper, Phys. Rev. B 54 (1996) 15742-15746. doi:10.1103/PhysRevB.54.15742. URL http://link.aps.org/doi/10.1103/PhysRevB.54.15742

[17] M. M. G. Alemany, C. Rey, L. J. Gallego, Transport coefficients of liquid transition metals: A computer simulation study using the embedded atom model, The Journal of Chemical Physics 109 (13) (1998) 5175-5176. doi:10.1063/1.477133.

[18] A. Pasquarello, K. Laasonen, R. Car, C. Lee, D. Vanderbilt, Ab initio molecular dynamics for $d$-electron systems: Liquid copper at 1500 k, Phys. Rev. Lett. 69 (1992) 1982-1985. doi:10.1103/PhysRevLett.69.1982. URL http://link.aps .org/doi/10.1103/PhysRevLett.69.1982

[19] A. D. Cicco, A. Trapananti, Study of local icosahedral ordering in liquid and undercooled liquid copper, Journal of NonCrystalline Solids 353 (32-40) (2007) 3671 - 3678. doi:http://dx.doi.org/10.1016/j.jnoncrysol.2007.05.150. URL http://www.sciencedirect.com/science/article/pii/S0022309307006473

[20] M. Celino, V. Rosato, A. Di Cicco, A. Trapananti, C. Massobrio, Role of defective icosahedra in undercooled copper, Phys. Rev. B 75 (2007) 174210. doi:10.1103/PhysRevB.75.174210. URL http://link.aps.org/doi/10.1103/PhysRevB.75.174210

[21] M. Celino, Molecular dynamics characterization of icosahedral short range order in undercooled copper, The European Physical Journal Special Topics 196 (1) (2011) 35-43. doi:10.1140/epjst/e2011-01416-1. URL http://dx.doi.org/10.1140/epjst/e2011-01416-1

[22] P. Ganesh, M. Widom, Signature of nearly icosahedral structures in liquid and supercooled liquid copper, Phys. Rev. B 74 (2006) 134205. doi:10.1103/PhysRevB.74.134205. URL http://link. aps.org/doi/10.1103/PhysRevB.74.134205

[23] G. Voronoi, J. Reine Angew. Math. 134 (1908) 199.

[24] S.-P. Pan, S.-D. Feng, J.-W. Qiao, W.-M. Wang, J.-Y. Qin, Crystallization pathways of liquid-bcc transition for a model iron by fast quenching, Scientific Reports 5 (2015) 16956. doi:10.1038/srep16956. 
[25] W. M. C. Foulkes, R. Haydock, Tight-binding models and density-functional theory, Phys. Rev. B 39 (1989) 12520-12536. doi:10.1103/PhysRevB.39.12520.

[26] P. Pulay, Ab initio calculation of force constants and equilibrium geometries in polyatomic molecules, Molecular Physics 17 (2) (1969) 197-204. doi:10.1080/00268976900100941.

[27] M. Kabir, A. Mookerjee, A. K. Bhattacharya, Structure and stability of copper clusters: A tight-binding molecular dynamics study, Phys. Rev. A 69 (2004) 043203. doi:10.1103/PhysRevA.69.043203. URL http://link.aps.org/doi/10.1103/PhysRevA.69.043203

[28] M. J. Mehl, D. A. Papaconstantopoulos, Topics in Computational Materials Science, World Scientific, Singapore, 1998, Ch. V, pp. 169-213.

[29] D. A. Papaconstantopoulos, M. J. Mehl, B. Akdim, Applications of a new tight-binding total energy method, in: B. K. Rao (Ed.), Proceedings of the International Symposium on Novel Materials, 1998, pp. 393-403.

[30] R. E. Cohen, M. J. Mehl, D. A. Papaconstantopoulos, Phys. Rev. B 50 (1994) 14694.

[31] M. J. Mehl, D. A. Papaconstantopoulos, N. Kioussis, M. Herbranson, Phys. Rev. B 61 (2000) 4894.

[32] F. Kirchhoff, M. J. Mehl, N. I. Papanicolaou, D. A. Papaconstantopoulos, F. S. Khan, Phys. Rev. B 63 (2001) 195101.

[33] Y. Mishin, M. J. Mehl, D. A. Papaconstantopoulos, A. F. Voter, J. D. Kress, Phys. Rev. B 63 (2001) 224106.

[34] D. A. Papaconstantopoulos, M. Lach-hab, M. J. Mehl, Physica B 296 (2001) 129.

[35] D. A. Papaconstantopoulos, M. J. Mehl, The Slater-Koster tight-binding method: a computationally efficient and accurate approach, Journal of Physics: Condensed Matter 15 (10) (2003) R413.

[36] A. Sutton, M. Finnis, D. Pettifor, Y. Ohta, The tight-binding bond model, Journal of Physics C - Solid State Physics 21 (1988) 35-66. URL http://dx.doi.org/10.1088/0022-3719/21/1/007

[37] M. J. Mehl, D. A. Papaconstantopoulos, R. E. Cohen, A tight-binding method for the evaluation of the total energy of large systems, International Journal of Thermophysics 16 (2) (1995) 503-510. doi:10.1007/BF01441916.

[38] J. Dziedzic, M. Bobrowski, J. Rybicki, Hybrid quantum-classical approach for atomistic simulation of metallic systems, Phys. Rev. B 83 (2011) 224114. doi:10.1103/PhysRevB.83.224114.

[39] J. Brillo, I. Egry, Density determination of liquid copper, nickel, and their alloys, International Journal of Thermophysics 24 (4) (2003) 1155-1170. doi:10.1023/A:1025021521945.

[40] J. Dziedzic, Quantum-classical calculations of the nanomechanical properties of metals, TASK Quarterly 13 (2009) 207310.

[41] S. Nosé, A unified formulation of the constant temperature molecular dynamics methods, The Journal of Chemical Physics 81 (1) (1984) 511-519. doi:http://dx.doi.org/10.1063/1.447334.

[42] W. G. Hoover, Canonical dynamics: Equilibrium phase-space distributions, Phys. Rev. A 31 (3) (1985) 1695-1697.

[43] A. C. Brańka, K. W. Wojciechowski, Generalization of Nosé and Nosé-Hoover isothermal dynamics, Phys. Rev. E 62 (3) (2000) 3281-3292.

[44] C. W. Gear, The automatic integration of ordinary differential equations, Commun. ACM 14 (3) (1971) 176-179. doi:10.1145/362566.362571. URL http://doi.acm.org/10.1145/362566.362571

[45] D. W. Qi, S. Wang, Icosahedral order and defects in metallic liquids and glasses, Phys. Rev. B 44 (1991) $884-887$. doi:10.1103/PhysRevB.44.884. URL http://link.aps.org/doi/10.1103/PhysRevB.44.884

[46] T. Fukunaga, K. Itoh, T. Otomo, K. Mori, M. Sugiyama, H. Kato, M. Hasegawa, A. Hirata, Y. Hirotsu, A. Hannon, Voronoi analysis of the structure of $\mathrm{Cu}-\mathrm{Zr}$ and $\mathrm{Ni}-\mathrm{Zr}$ metallic glasses, Intermetallics 14 (8-9) (2006) 893 - 897, Fourth International Conference on Bulk Metallic Glasses. doi:http://dx.doi.org/10.1016/j.intermet.2006.01.006. URL http://www.sciencedirect.com/science/article/pii/S0966979506000197

[47] L. Yang, S. Yin, X. D. Wang, Q. P. Cao, J. Z. Jiang, K. Saksl, H. Franz, Atomic structure in Zr70Ni30 metallic glass, Journal of Applied Physics 102 (8) (2007) 083512. doi:http://dx.doi.org/10.1063/1.2798386. URL http://scitation.aip.org/content/aip/journal/jap/102/8/10.1063/1.2798386

[48] P. Ganesh, M. Widom, Ab initio simulations of geometrical frustration in supercooled liquid $\mathrm{Fe}$ and Fe-based metallic glass, Phys. Rev. B 77 (2008) 014205. doi:10.1103/PhysRevB.77.014205. URL http://link. aps.org/doi/10.1103/PhysRevB.77.014205

[49] S. G. Hao, C. Z. Wang, M. J. Kramer, K. M. Ho, Microscopic origin of slow dynamics at the good glass forming composition range in $\mathrm{zr}_{1-x} \mathrm{cu}_{x}$ metallic liquids, Journal of Applied Physics 107 (5) (2010) 053511. doi:http://dx.doi.org/10.1063/1.3298896.

URL http://scitation.aip.org/content/aip/journal/jap/107/5/10.1063/1.3298896

[50] S. Wu, M. Kramer, X. Fang, S. Wang, C. Wang, K. Ho, Z. Ding, L. Chen, Icosahedral short-range order in amorphous Cu80Si20 by ab initio molecular dynamics simulation study, Intermetallics 30 (2012) 122 - 126, Bulk Metallic Glasses VIII. doi:http://dx.doi.org/10.1016/j.intermet.2012.03.018. URL http://www.sciencedirect.com/science/article/pii/S096697951200101X

[51] F. Li, X. Liu, Z. Lu, Atomic structural evolution during glass formation of a $\mathrm{Cu}-\mathrm{Zr}$ binary metallic glass, Computational Materials Science 85 (2014) 147 - 153. doi:http://dx.doi.org/10.1016/j.commatsci.2013.12.058.

URL http://www.sciencedirect.com/science/article/pii/s0927025614000032

[52] M. Shimono, H. Onodera, Dynamics and geometry of icosahedral order in liquid and glassy phases of metallic glasses, Metals 5 (3) (2015) 1163. doi:10.3390/met5031163.

URL http://www . mdpi . com/2075-4701/5/3/1163 

Computational Materials Science 114 (2016) 219 - 232. doi:https://doi.org/10.1016/j.commatsci.2015.12.014 URL http://www.sciencedirect.com/science/article/pii/s0927025615007909

[54] M. Leocmach, H. Tanaka, Roles of icosahedral and crystal-like order in the hard spheres glass transition, Nature Communications 3 (2012) 974. doi:10.1038/ncomms1974. URL http://dx.doi .org/10.1038/ncomms1974

[55] J. D. Honeycutt, H. C. Andersen, Molecular dynamics study of melting and freezing of small Lennard-Jones clusters, The Journal of Physical Chemistry 91 (19) (1987) 4950-4963. doi:10.1021/j100303a014. URL http://dx.doi.org/10.1021/j100303a014

[56] F. F. Chen, H. F. Zhang, F. X. Qin, Z. Q. Hu, Molecular dynamics study of atomic transport properties in rapidly cooling liquid copper, The Journal of Chemical Physics 120 (4) (2004) 1826-1831. doi:http://dx.doi.org/10.1063/1.1636452. URL http://scitation.aip.org/content/aip/journal/jcp/120/4/10.1063/1.1636452

[57] U. R. Pedersen, T. B. Schrøder, J. C. Dyre, P. Harrowell, Geometry of slow structural fluctuations in a supercooled binary alloy, Phys. Rev. Lett. 104 (2010) 105701. doi:10.1103/PhysRevLett.104.105701. URL http://link.aps.org/doi/10.1103/PhysRevLett.104.105701

[58] Z.-C. Xie, T.-H. Gao, X.-T. Guo, X.-M. Qin, Q. Xie, Network connectivity in icosahedral medium-range order of metallic glass: A molecular dynamics simulation, Journal of Non-Crystalline Solids 406 (2014) $31-36$. doi:http://dx.doi.org/10.1016/j.jnoncrysol.2014.09.043 URL http://www.sciencedirect.com/science/article/pii/s0022309314004980

[59] N. Jakse, A. Pasturel, Stokes-Einstein relation and excess entropy scaling law in liquid copper, Condensed Matter Physics 18 (4) (2015) 43603. doi:10.5488/CMP.18.43603.

[60] A. S. Clarke, H. Jónsson, Structural changes accompanying densification of random hard-sphere packings, Phys. Rev. E 47 (1993) 3975-3984. doi:10.1103/PhysRevE.47.3975. URL http://link.aps.org/doi/10.1103/PhysRevE.47.3975

[61] D. Faken, H. Jónsson, Systematic analysis of local atomic structure combined with 3D computer graphics, Computational Materials Science 2 (2) (1994) 279 - 286. doi:http://dx.doi.org/10.1016/0927-0256(94)90109-0. URL http://www.sciencedirect.com/science/article/pii/0927025694901090

[62] M. Liška, P. Perichta, B. Hatalová, The structure of molecular dynamics simulated oxide glasses viewed through Voronoi polyhedra tesselation, Journal of Non-Crystalline Solids 192 (1995) 249 - 252. doi:http://dx.doi.org/10.1016/00223093(95)00358-4. URL http://www.sciencedirect.com/science/article/pii/0022309395003584

[63] W. Brostow, M. Chybicki, R. Laskowski, J. Rybicki, Voronoi polyhedra and Delaunay simplexes in the structural analysis of molecular-dynamics-simulated materials, Phys. Rev. B 57 (1998) 13448-13458. doi:10.1103/PhysRevB.57.13448. URL http://link.aps.org/doi/10.1103/PhysRevB.57.13448

[64] C. Chakravarty, P. G. Debenedetti, F. H. Stillinger, Generating inherent structures of liquids: Comparison of local minimization algorithms, The Journal of Chemical Physics 123 (20) (2005) 206101. doi:http://dx.doi.org/10.1063/1.2129327. URL http://scitation.aip.org/content/aip/journal/jcp/123/20/10.1063/1.2129327

[65] S. N. Chakraborty, C. Chakravarty, Diffusivity, excess entropy, and the potential-energy landscape of monatomic liquids, The Journal of Chemical Physics 124 (1) (2006) 014507. doi:http://dx.doi.org/10.1063/1.2140282. URL http://scitation.aip.org/content/aip/journal/jcp/124/1/10.1063/1.2140282

[66] P. M. Larsen, S. Schmidt, J. Schiøtz, Robust structural identification via polyhedral template matching, Modelling and Simulation in Materials Science and Engineering 24 (5) (2016) 055007. doi:10.1088/0965-0393/24/5/055007. URL https://doi.org/10.1088/0965-0393/24/5/055007

[67] Y. Wang, S. Teitel, C. Dellago, Surface-driven bulk reorganization of gold nanorods, Nano Letters 5 (11) (2005) $2174-2178$. doi:10.1021/nl051149h. URL http://dx.doi.org/10.1021/n1051149h

[68] Y. Wang, S. Teitel, C. Dellago, Effect of surface structure on shape transformations of gold nanorods, Journal of Computational and Theoretical Nanoscience 4 (2) (2007) 282-290. doi:doi:10.1166/jctn.2007.013. URL http://www.ingentaconnect.com/content/asp/jctn/2007/00000004/00000002/art00013

[69] W. Lechner, C. Dellago, Accurate determination of crystal structures based on averaged local bond order parameters, The Journal of Chemical Physics 129 (11) (2008) 114707. doi:http://dx.doi.org/10.1063/1.2977970. URL http://scitation.aip.org/content/aip/journal/jcp/129/11/10.1063/1.2977970

[70] J. S. van Duijneveldt, D. Frenkel, Computer simulation study of free energy barriers in crystal nucleation, The Journal of Chemical Physics 96 (6) (1992) 4655-4668. doi:http://dx.doi.org/10.1063/1.462802. URL http://scitation.aip.org/content/aip/journal/jcp/96/6/10.1063/1.462802

[71] P. R. ten Wolde, M. J. Ruiz-Montero, D. Frenkel, Numerical evidence for bcc ordering at the surface of a critical fcc nucleus, Phys. Rev. Lett. 75 (1995) 2714-2717. doi:10.1103/PhysRevLett.75.2714. URL http://link.aps .org/doi/10.1103/PhysRevLett.75.2714

[72] I. Volkov, M. Cieplak, J. Koplik, J. R. Banavar, Molecular dynamics simulations of crystallization of hard spheres, Phys. Rev. E 66 (2002) 061401. doi:10.1103/PhysRevE.66.061401. URL http://link.aps.org/doi/10.1103/PhysRevE.66.061401

[73] C. Chakravarty, R. M. Lynden-Bell, Landau free energy curves for melting of quantum solids, The Journal of Chemical Physics 113 (20) (2000) 9239-9247. doi:http://dx.doi.org/10.1063/1.1316105. URL http://scitation.aip.org/content/aip/journal/jcp/113/20/10.1063/1.1316105

[74] C. Chakravarty, P. G. Debenedetti, F. H. Stillinger, Lindemann measures for the solid-liquid phase transition, The Journal 
of Chemical Physics 126 (20) (2007) 204508. doi:http://dx.doi.org/10.1063/1.2737054.

URL http://scitation.aip.org/content/aip/journal/jcp/126/20/10.1063/1.2737054

[75] R. Lynden-Bell, J. Van Duijneveldt, D. Frenkel, Free energy changes on freezing and melting ductile metals, Molecular Physics 80 (4) (1993) 801-814. doi:10.1080/00268979300102661.

URL http://dx.doi.org/10.1080/00268979300102661

[76] W. Mickel, S. C. Kapfer, G. E. Schröder-Turk, K. Mecke, Shortcomings of the bond orientational order parameters for the analysis of disordered particulate matter, The Journal of Chemical Physics 138 (4) (2013) 044501. doi:http://dx.doi.org/10.1063/1.4774084.

URL http://scitation.aip.org/content/aip/journal/jcp/138/4/10.1063/1.4774084

[77] A. Stukowski, Structure identification methods for atomistic simulations of crystalline materials, Modelling and Simulation in Materials Science and Engineering 20 (4) (2012) 045021.

URL http://stacks . iop. org/0965-0393/20/i=4/a=045021

[78] C. H. Rycroft, VORO ++: A three-dimensional Voronoi cell library in C++, Chaos 19 (4) (2009) 041111. doi:http://dx.doi.org/10.1063/1.3215722.

URL http://scitation.aip.org/content/aip/journal/chaos/19/4/10.1063/1.3215722

[79] S. Winczewski, J. Dziedzic, J. Rybicki, A highly-efficient technique for evaluating bond-orientational order parameters, Computer Physics Communications 198 (2016) 128 - 138. doi:http://dx.doi.org/10.1016/j.cpc.2015.09.009.

URL http://www.sciencedirect.com/science/article/pii/S0010465515003471

[80] A. Stukowski, Visualization and analysis of atomistic simulation data with OVITO - the Open Visualization Tool, Modelling and Simulation in Materials Science and Engineering 18 (1) (2010) 015012. URL http://stacks. iop.org/0965-0393/18/i=1/a=015012

[81] P. N. Pusey, W. van Megen, Observation of a glass transition in suspensions of spherical colloidal particles, Phys. Rev. Lett. 59 (1987) 2083-2086. doi:10.1103/PhysRevLett.59.2083 URL http://link.aps.org/doi/10.1103/PhysRevLett.59.2083

[82] J. Park, Y. Shibutani, Weighted Voronoi tessellation technique for internal structure of metallic glasses, Intermetallics 15 (2) (2007) 187 - 192. doi:https://doi.org/10.1016/j.intermet.2006.05.005. URL http://www.sciencedirect.com/science/article/pii/S0966979506001890

[83] M. Wakeda, Y. Shibutani, Icosahedral clustering with medium-range order and local elastic properties of amorphous metals, Acta Materialia 58 (11) (2010) 3963 - 3969. doi:https://doi.org/10.1016/j.actamat.2010.03.029. URL http://www.sciencedirect.com/science/article/pii/S1359645410001746

[84] B. Lu, L. Kong, K. Laws, W. Xu, Z. Jiang, Y. Huang, M. Ferry, J. Li, Y. Zhou, EXAFS and molecular dynamics simulation studies of $\mathrm{Cu}-\mathrm{Zr}$ metallic glass: Short-to-medium range order and glass forming ability, Materials Characterization 141 (2018) $41-48$. doi:https://doi.org/10.1016/j.matchar.2018.04.036.

URL http://www.sciencedirect.com/science/article/pii/S1044580318302870 